\title{
Estimation of $P(X<Y)$ using Some Modifications of Ranked Set Sampling for Weibull Distribution
}

\author{
Fatma Gül Akgül \\ Department of Computer Engineering, Artvin Çoruh University, Turkey \\ ftm.gul.fuz@artvin.edu.tr \\ Birdal Şenoğlu \\ Department of Statistics, Ankara University, Turkey \\ senoglu@science.ankara.edu.tr
}

\begin{abstract}
In statistical literature, estimation of $R=P(X<Y)$ is a commonly-investigated problem, and consequently, there have been considerable number of studies dealing with its estimation of it under simple random sampling (SRS). However, in recent years, the ranked set sampling (RSS) method have been widely-used in the estimation of $R$. In this study, we consider the estimation of $R$ when the distribution of the both stress and strength are Weibull under the modification of RSS, which are extreme ranked set sampling (ERSS), median ranked set sampling (MRSS) and percentile ranked set sampling (PRSS). We obtain the estimators of $R$ using the maximum likelihood (ML) and the modified maximum likelihood (MML) methodologies under these modifications. Then the performances of proposed estimators are compared with the corresponding ML and MML estimators of $R$ using SRS via a Monte-Carlo simulation study.
\end{abstract}

Keywords: Stress-strength model, Extreme ranked set sampling, Median ranked set sampling, Percentile ranked set sampling, Efficiency.

\section{Introduction}

In the context of reliability, making statistical inferences concerning $R=P(X<Y)$ has received considerable attention in the literature. In this sense, $Y$ represents the strength of a system which is subject to stress $X$. It is clear that, the system fails when stress exceeds strength. Therefore, the stress-strength model $R=P(X<Y)$ is a measure of system reliability. In general, the problem of the estimation of $R$ is studied under SRS data. For example, Church and Harris (1970), Tong (1977), Constantine et al. (1986) and Kundu and Gupta (2006) all discuss this problem when $X$ and $Y$ are two independent normal, exponential, gamma and Weibull random variables; for more detailed information, see also Kotz et al. (2003). Nevertheless, in recent years, several authors have considered the estimation of $R$ based ranked set sampling (RSS) using both parametric and non-parametric methods. For example, Sengupta and Mukhuti (2008a, b), Mutlak et al. (2010), Dong et al. (2013) and Akgül and Şenoğlu (2017) tackled this problem using parametric methods. Mahdizadeh and Zamanzade (2016a,b, 2017), Dastbaravarde and Zamanzadeh (2017) discussed this problem using non-parametric methods.

The RSS method was first proposed by McIntyre (1952) as a cost-effective and more structural alternative approach to SRS. In RSS procedure, without any certain measurement, sampling units can be ranked easily and cheaply with respect to characteristic of interest. The efficiency of RSS according to SRS in different statistical methods has been investigated by several researchers, see Takahasi and Wakimoto (1968), Chen et al. (2004) and Noughabi (2017). 
While sampling units are constituted in the RSS method, due to not doing certain measurements, the possibility of doing error in ranking increases. In order to overcome this problem, various modifications of RSS have been suggested; see Samawi et al. (2017), such as extreme ranked set sampling, ERSS (Samavi et al., 1996), median ranked set sampling, MRSS (Muttlak, 1997), double ranked set sampling, DRSS (Al-Saleh and AlKadiri, 2000), percentile ranked set sampling, PRSS (Muttlak, 2003), L ranked set sampling, LRSS (Al-Nasser, 2007) and neoteric ranked set sampling, NRSS (Zamanzade and Al-Omari, 2016). Besides these studies, several authors have considered the estimation of the parameters of well-known distributions using RSS or modifications of it. For example, the estimation of unknown parameters of exponential, extreme-value, logistic, Weibull and Pareto distributions was studied by Lam et al. (1994), Bhoj (1997), AbuDayyeh et al. (2004), Helu et al. (2010) and Abu-Dayyeh et al. (2013). Also, Shaibu and Muttlak $(2002,2004)$ were based on RSS and its modifications.

In this paper, we estimate system reliability $R$ under the ERSS, MRSS and PRSS methods where stress $X \sim W \operatorname{eibull}\left(p, \sigma_{1}\right)$ and the strength $Y \sim W \operatorname{eibull}\left(p, \sigma_{2}\right)$ are both independent. The reason for using these particular modifications is for illustration and brevity. Other modifications may also be conducted in a similar manner.

The probability density function (pdf) and the cumulative density function (cdf) of two parameter Weibull distribution with scale parameter $\sigma$ and shape parameter $p$ are given as shown below

$$
f(x, p, \sigma)=\frac{p}{\sigma} x^{p-1} e^{-\frac{x^{p}}{\sigma}}, x>0, p>0, \sigma>0
$$

and

$$
F(x, p, \sigma)=1-e^{-\frac{x^{p}}{\sigma}}, x>0, p>0, \sigma>0
$$

respectively. Using these pdf and cdf, it is easy to obtain that the system reliability $R$ is derived as follows

$$
\begin{aligned}
R=P(X<Y) & =\int_{0}^{\infty} F_{X}(t) f_{y}(t) d t \\
& =\int_{0}^{\infty}\left(1-e^{-\frac{t^{p}}{\sigma_{1}}}\right) \frac{p}{\sigma_{2}} t^{p-1} e^{-\frac{t^{p}}{\sigma_{2}}} d t \\
& =\frac{\sigma_{2}}{\sigma_{1}+\sigma_{2}} .
\end{aligned}
$$

Similar to Akgül and Şenoğlu (2014) and Akgül (2015), we obtain the ML estimators of $R$ using two different approaches. First, the likelihood equations are solved using numerical methods. Second, we solve the likelihood equations by using the methodology called as modified maximum likelihood (MML), as proposed by Tiku $(1967,1968)$. This is based on the idea of linearization of non-linear terms in likelihood equations by using the Taylor series expansion around the expected values of the standardized order statistics. We then compare the performances of the proposed estimators with the corresponding estimators of $R$ based on SRS using an extensive Monte Carlo simulation study. Mean square error 
(MSE) criterion is used in comparisons to determine the most efficient estimator and sampling method.

The rest of the paper is organized as follows: In Section 2, we give brief descriptions regarding modifications of RSS. We obtain the ML estimators of $R$ based on ERSS, MRSS and PRSS in Section 3. In Section 4, we derive the MML estimators of $R$ based on the modifications of RSS. Section 5 is devoted to the Monte-Carlo simulation study. Comments and conclusions are given in the final section.

\section{Descriptions of the modifications}

In this section, we give brief descriptions of the ERSS, MRSS and PRSS methods. Definitions of notations frequently used in this section and in the rest of the paper are given as shown follows

$m$ : set sizes,

$r$ : number of cycles,

$n$ : sample sizes.

\subsection{ERSS Method}

ERSS, first proposed by Samawi et al. (1996), is more efficient than SRS when underlying distribution is symmetric. In addition, the usage of ERSS is more practical than the usage of RSS, because we are only interested in selecting the smallest and largest observations and measuring them.

We now present the procedure of ERSS as shown below

Step 1. Select $m$ random sets via SRS, each of size $m$, called as a cycle.

Step 2. Rank the units with respect to variable of interest by virtual comparisons, expert opinion or axillary variables.

Step 3. If set size $m$ is odd,

i. $\quad$ First, the smallest ranked unit is selected from the first $(m-1) / 2$ sets.

ii. Then, the median ranked unit is selected from the next set.

iii. Finally, the largest ranked unit is selected from the second $(m-1) / 2$ sets. If set size $m$ is even,

$i$. The smallest ranked unit is selected from the first $m / 2$ sets.

ii. Then, the largest ranked unit is selected from the second $m / 2$ sets.

Step 4. Repeat Steps 1-3 $r$ times, until the sample size $n=m r$ is obtained.

\subsection{MRSS Method}

The MRSS method was proposed by Muttlak (1997) to reduce the ranking error which is encountered in the RSS method. It also increases the efficiency of the estimator for both perfect and imperfect ranking.

The procedure of MRSS is exactly the same as the procedure of ERSS except for Step 3. Therefore, we just give the definition of Step 3 for brevity: 
Step 3. If set size $m$ is odd,

$i$. The median ranked unit is selected from each ordered set.

If set size $m$ is even,

$i$. The $(m / 2)$ th smallest ranked unit is selected from the first $m / 2$ sets.

ii. Then, $(m / 2+1)$ th smallest ranked unit is selected from the second $m / 2$ sets.

\subsection{PRSS Method}

The PRSS method was proposed by Muttlak (2003) to estimate the population mean. This method has practical usage according to RSS, since we only select and measure the $p t h$ and $q$ th percentile of the sample. Here, $0<p<1$ and $q=1-p$. Therefore, the PRSS method reduces the ranking error compared to the RSS method.

The procedure for the PRSS method is summarized as follows:

From the same reasons given in subsection 2.2, we will define the Step 3.

Step 3. If set size $m$ is odd,

i. First, the $(p(m+1))$ th the smallest ranked unit is selected from the first $(m-1) / 2$ sets

ii. Then, the median ranked unit is selected from the following set.

iii. Finally, the $(q(m+1))$ th the smallest ranked unit is selected from the second $(m-1) / 2$ sets.

If set size $m$ is even,

i. $\quad$ The $(p(m+1))$ th smallest ranked unit is selected from the first $(m / 2)$ sets.

ii. The $(q(m+1))$ th the smallest ranked unit is selected from the second $(m / 2)$ sets.

Here, it should be noted that $(p(m+1))$ and $(q(m+1))$ represent the nearest integer value of $p(m+1)$ and $q(m+1)$, respectively.

\section{ML Estimators of $\boldsymbol{R}$}

In this section, we obtain the ML estimators of $R$ based on the modifications of RSS namely ERSS, MRSS and PRSS. As earlier stated, the stress $X \sim W \operatorname{Wibull}\left(p, \sigma_{1}\right)$ and the strength $Y \sim W e i b u l l\left(p, \sigma_{2}\right)$ are both independent. We also give a number of useful notations to be used throughout the paper as shown below:

$m_{x}$ and $m_{y}$ : the set sizes corresponding to $X$ and $Y$, respectively.

$r_{x}$ and $r_{y}$ : the number of cycles corresponding to $X$ and $Y$, respectively.

$n$ and $m$ : sample sizes corresponding to $X$ and $Y$, respectively. 
First of all, we obtain the likelihood equations for estimating the scale parameters $\sigma_{1}$ and $\sigma_{2}$ and as well as the shape parameter $p$ for the modifications of RSS separately.

\section{Likelihood equations for ERSS:}

Case 1: Odd set sizes.

Let

$X_{(1) i c}, i=1, \ldots, \frac{m_{x}-1}{2} \cup X_{\left(\frac{m_{x}+1}{2}\right) i c}, i=\frac{m_{x}+1}{2} X_{\left(m_{x}\right) i c}, i=\frac{m_{x}+1}{2}+1, \ldots m_{x} ; c=1, \ldots, r_{x}$

and

$Y_{(1) j l}, j=1, \ldots, \frac{m_{y}-1}{2} \cup Y_{\left(\frac{m_{y}+1}{2}\right) j l}, j=\frac{m_{y}+1}{2} \cup Y_{\left(m_{y}\right) j l}, j=\frac{m_{y}+1}{2}+1, \ldots m_{y} ; l=1, \ldots, r_{y}$

be ERSS samples. Here, let $X_{(1) i c}, X_{\left(\frac{m_{x}+1}{2}\right) i c}$ and $X_{\left(m_{x}\right) i c}$ be the smallest, median and largest order statistics from the $i$ th set of size $m_{x}$ of the $c$ th cycle, respectively. Similarly, let $Y_{(1) j l}, Y_{\left(\frac{m_{y}+1}{2}\right) j l}$ and $Y_{\left(m_{y}\right) j l}$ be the smallest, median and largest order statistics from the $j$ th set of size $m_{y}$ of the $l$ th cycle, respectively.

Then, the likelihood function of the ERSS is shown below

$$
\begin{aligned}
L & =\prod_{c=1}^{r_{x}} \prod_{i=1}^{\frac{m_{x}-1}{2}} f_{1: m_{x}}\left(x_{(1) i c}\right) \prod_{c=1}^{r_{x}} f_{\frac{m_{x}+1}{2} m_{x}}\left(x_{\left(\frac{m_{x}+1}{2}\right) \frac{m_{x}+1}{2}}\right) \prod_{c=1}^{r_{x}} \prod_{i=\frac{m_{x}+1}{2}+1}^{m_{x}} f_{m_{x}: m_{x}}\left(x_{\left(m_{x}\right) i c}\right) \\
& \cdot \prod_{l=1}^{r_{y}} \prod_{j=1}^{\frac{m_{y}-1}{2}} f_{1: m_{y}}\left(y_{(1) j l}\right) \prod_{l=1}^{r_{y}} f_{\frac{m_{y}+1}{2}: m_{y}}\left(y_{\left.\left(\frac{m_{y}+1}{2}\right) \frac{m_{y}+1}{2}\right)}\right) \prod_{l=1}^{r_{y}} \prod_{j=\frac{m_{y}+1}{2}+1}^{m_{y}} f_{m_{y}: m_{y}}\left(y_{\left(m_{y}\right) j l}\right)(4)
\end{aligned}
$$

where

$$
\begin{aligned}
& f_{1: m_{x}}\left(x_{(1)}\right)=m_{x} f\left(x_{(1)}\right)\left[1-F\left(x_{(1)}\right)\right]^{m_{x}-1}, \\
& f_{m_{x}: m_{x}}\left(x_{\left(m_{x}\right)}\right)=m_{x} f\left(x_{\left(m_{x}\right)}\right)\left[F\left(x_{\left(m_{x}\right)}\right)\right]^{m_{x}-1}, \\
& f_{\frac{m_{x}+1}{2}: m_{x}}\left(x_{\left(\frac{m_{x}+1}{2}\right)}\right)=\frac{m_{x} !}{\left(\left(\frac{m_{x}-1}{2}\right) !\right)^{2}} f\left(x_{\left(\frac{m_{x}+1}{2}\right)}\right)\left[F\left(x_{\left(\frac{m_{x}+1}{2}\right)}\right)\right]^{\frac{m_{x}-1}{2}}\left[1-F\left(x_{\left(\frac{m_{x}+1}{2}\right)}\right)\right]^{\frac{m_{x}-1}{2}},
\end{aligned}
$$

see equations (1) and (2) for $f(x)$ and $F(x)$. Similarly, $f_{1: m_{y}}\left(y_{(1)}\right), f_{m_{y}: m_{y}}\left(y_{\left(m_{y}\right)}\right)$ and $f_{\frac{m_{y}+1}{2}: m_{y}}\left(y_{\left(\frac{m_{y}+1}{2}\right)}\right)$ are defined as in (5), (6) and (7), respectively.

The ML estimators of the unknown parameters $p, \sigma_{1}$ and $\sigma_{2}$ are the solutions of the following likelihood equations: 


$$
\begin{aligned}
& \frac{\partial \ln L}{\partial p}=\frac{n+m}{p}+\sum_{c=1}^{r_{x}} \sum_{i=1}^{m^{\prime}} \ln x_{(1) i c}-\frac{m_{x}}{\sigma_{1}} \sum_{c=1}^{r_{x}} \sum_{i=1}^{m^{\prime}} x_{(1) i c}^{p} \ln x_{(1) i c}+\sum_{c=1}^{r_{x}} \sum_{i=m^{\prime}+2}^{m_{x}} \ln x_{\left(m_{x}\right) i c} \\
& -\frac{1}{\sigma_{1}} \sum_{c=1}^{r_{x}} \sum_{i=m^{\prime}+2}^{m_{x}} x_{\left(m_{x}\right) i c}^{p} \ln x_{\left(m_{x}\right) i c}+\frac{m_{x}-1}{\sigma_{1}} \sum_{c=1}^{r_{x}} \sum_{i=m^{\prime}+2}^{m_{x}} \frac{x_{\left(m_{x}\right) i c}^{p} \ln x_{\left(m_{x}\right) i c}}{e^{x_{\left(m_{x}\right) i c}^{p} / \sigma_{1}}-1} \\
& +\sum_{l=1}^{r_{y}} \sum_{j=1}^{m^{\prime \prime}} \ln y_{(1) j l}-\frac{m_{y}}{\sigma_{2}} \sum_{l=1}^{r_{y}} \sum_{j=1}^{m^{\prime \prime}} y_{(1) j l}^{p} \ln y_{(1) j l}+\sum_{l=1}^{r_{y}} \sum_{j=m^{\prime \prime}+2}^{m_{y}} \ln y_{\left(m_{y}\right) j l} \\
& -\frac{1}{\sigma_{2}} \sum_{l=1}^{r_{y}} \sum_{j=m^{\prime \prime}+2}^{m_{y}} y_{\left(m_{y}\right) j l}^{p} \ln y_{\left(m_{y}\right) j l}+\frac{m_{y}-1}{\sigma_{2}} \sum_{l=1}^{r_{y}} \sum_{j=m^{\prime \prime}+2}^{m_{y}} \frac{y_{\left(m_{y}\right) j l}^{p} \ln y_{\left(m_{y}\right) j l}}{e^{y_{\left(m_{y}\right) j l}^{p} / \sigma_{2}}-1} \\
& +\sum_{c=1}^{r_{x}} \ln x_{\left(m^{\prime}+1\right) m^{\prime}+1 c}-\frac{m^{\prime}+1}{\sigma_{1}} \sum_{c=1}^{r_{x}} x_{\left(m^{\prime}+1\right) m^{\prime}+1 c}^{p} \ln x_{\left(m^{\prime}+1\right) m^{\prime}+1 c} \\
& +\frac{m^{\prime}}{\sigma_{1}} \sum_{c=1}^{r_{x}} \frac{x_{\left(m^{\prime}+1\right) m^{\prime}+1 c}^{p} \ln x_{\left(m^{\prime}+1\right) m^{\prime}+1 c}}{e^{x_{\left(m^{\prime}+1\right) m^{\prime}+1 c}^{p} / \sigma_{1}}-1}+\sum_{l=1}^{r_{y}} \ln y_{\left(m^{\prime \prime}+1\right) m^{\prime \prime}+1 l} \\
& -\frac{m^{\prime \prime}+1}{\sigma_{2}} \sum_{l=1}^{r_{y}} y_{\left(m^{\prime \prime}+1\right) m^{\prime \prime}+1 l}^{p} \ln y_{\left(m^{\prime \prime}+1\right) m^{\prime \prime}+1 l} \\
& +\frac{m^{\prime \prime}}{\sigma_{2}} \sum_{l=1}^{r_{y}} \frac{y_{\left(m^{\prime \prime}+1\right) m^{\prime \prime}+1 l}^{p} \ln y_{\left(m^{\prime \prime}+1\right) m^{\prime \prime}+1 l}}{e^{y_{\left(m^{\prime \prime}+1\right) m^{\prime \prime}+1 l}^{p} / \sigma_{2}}-1}=0, \\
& \frac{\partial \ln L}{\partial \sigma_{1}}=-\frac{n}{\sigma_{1}}+\frac{m_{x}}{\sigma_{1}^{2}} \sum_{c=1}^{r_{x}} \sum_{i=1}^{m^{\prime}} x_{(1) i c}^{p}+\frac{1}{\sigma_{1}^{2}} \sum_{c=1}^{r_{x}} \sum_{i=m^{\prime}+2}^{m_{x}} x_{\left(m_{x}\right) i c}^{p} \\
& -\frac{m_{x}-1}{\sigma_{1}^{2}} \sum_{c=1}^{r_{x}} \sum_{i=m^{\prime}+2}^{m_{x}} \frac{x_{\left(m_{x}\right) i c}^{p}}{e^{x_{\left(m_{x}\right) i c}^{p} / \sigma_{1}}-1}+\frac{m^{\prime}+1}{\sigma_{1}^{2}} \sum_{c=1}^{r_{x}} x_{\left(m^{\prime}+1\right) m^{\prime}+1 c}^{p} \\
& -\frac{m^{\prime}}{\sigma_{1}^{2}} \sum_{c=1}^{r_{x}} \frac{x_{\left(m^{\prime}+1\right) m^{\prime}+1 c}^{p}}{e^{x_{\left(m^{\prime}+1\right) m^{\prime}+1 c}^{p} / \sigma_{1}}-1}=0, \\
& \frac{\partial \ln L}{\partial \sigma_{2}}=-\frac{m}{\sigma_{2}}+\frac{m_{y}}{\sigma_{2}^{2}} \sum_{l=1}^{r_{y}} \sum_{j=1}^{m^{\prime \prime}} y_{(1) j l}^{p}+\frac{1}{\sigma_{2}^{2}} \sum_{l=1}^{r_{y}} \sum_{j=m^{\prime \prime}+2}^{m_{y}} y_{\left(m_{y}\right) j l}^{p} \\
& -\frac{m_{y}-1}{\sigma_{2}^{2}} \sum_{l=1}^{r_{y}} \sum_{j=m^{\prime \prime}+2}^{m_{y}} \frac{y_{\left(m_{y}\right) j l}^{p}}{e^{y_{\left(m_{y}\right) j l}^{p} / \sigma_{2}}-1}+\frac{m^{\prime \prime}+1}{\sigma_{2}^{2}} \sum_{l=1}^{r_{y}} y_{\left(m^{\prime \prime}+1\right) m^{\prime \prime}+1 l}^{p} \\
& -\frac{m^{\prime \prime}}{\sigma_{2}^{2}} \sum_{l=1}^{r_{y}} \frac{y_{\left(m^{\prime \prime}+1\right) m^{\prime \prime}+1 l}^{p}}{e^{y_{\left(m^{\prime \prime}+1\right) m^{\prime \prime}+1 l^{\prime}}^{p} / \sigma_{2}}-1}=0
\end{aligned}
$$


Case 2. Even set sizes.

Let and

$$
X_{(1) i c}, i=1, \ldots, \frac{m_{x}}{2} \cup X_{\left(m_{x}\right) i c}, i=\frac{m_{x}}{2}+1, \ldots m_{x} ; c=1, \ldots, r_{x}
$$

$$
Y_{(1) j l}, j=1, \ldots, \frac{m_{y}}{2} \cup Y_{\left(m_{y}\right) j l}, j=\frac{m_{y}}{2}+1, \ldots m_{y} ; l=1, \ldots, r_{y}
$$

be ERSS samples. Here, $X_{(1) i c}, X_{\left(m_{x}\right) i c}, Y_{(1) j l}$ and $Y_{\left(m_{y}\right) j l}$ are defined as in Case 1. Then the likelihood function based on ERSS is given by

$$
\begin{aligned}
L= & \prod_{c=1}^{r_{x}} \prod_{i=1}^{\frac{m_{x}}{2}} f_{1: m_{x}}\left(x_{(1) i c}\right) \prod_{c=1}^{r_{x}} \prod_{i=\frac{m_{x}}{2}+1}^{m_{x}} f_{m_{x}: m_{x}}\left(x_{\left(m_{x}\right) i c}\right) \\
& \cdot \prod_{l=1}^{r_{y}} \prod_{j=1}^{\frac{m_{y}}{2}} f_{1: m_{y}}\left(y_{(1) j l}\right) \prod_{l=1}^{r_{y}} \prod_{j=\frac{m_{y}}{2}+1}^{m_{y}} f_{m_{y}: m_{y}}\left(y_{\left(m_{y}\right) j l}\right) .
\end{aligned}
$$

See Case 1 for the definitions of $f_{1: m_{x}}\left(x_{(1) i c}\right), f_{m_{x}: m_{x}}\left(x_{\left(m_{x}\right) i c}\right), f_{1: m_{y}}\left(y_{(1) j l}\right)$ and $f_{m_{y}: m_{y}}\left(y_{\left(m_{y}\right) j l}\right)$.

REMARK: It should be noted that we do not give the likelihood equations here and in the rest of the paper to avoid repetition. Therefore, we only give the likelihood functions for the sampling procedure ERSS, MRSS and PRSS when the set sizes are odd and even. The only exception is Section 4 (i.e., ERSS procedure, Case 1). The solutions of the likelihood equations are given at the end of this section for the modifications of RSS, since the likelihood equations for ERSS, MRSS and PRSS are more or the less the same in each case. The main objective for doing this is for the sake of brevity. Likelihood equations for each modification and case are available upon request from the first author of this paper.

\section{Likelihood functions for MRSS:}

Case 1. Odd set sizes.

Let

$$
X_{\left(\frac{m_{x}+1}{2}\right) i c^{\prime}} i=1, \ldots, m_{x}, c=1, \ldots, r_{x} \text { and } Y_{\left(\frac{m_{y}+1}{2}\right) j l}, j=1, \ldots, m_{y}, l=1, \ldots, r_{y}
$$

be the MRSS samples. Then the likelihood function is shown below

$$
L=\prod_{c=1}^{r_{x}} \prod_{i=1}^{m_{x}} f_{\frac{m_{x}+1}{2}: m_{x}}\left(x_{\left(\frac{m_{x}+1}{2}\right) i c}\right) \prod_{l=1}^{r_{y}} \prod_{j=1}^{m_{y}} f_{\frac{m_{y}+1}{2}: m_{y}}\left(y_{\left(\frac{m_{y}+1}{2}\right) j l}\right) .
$$

For the pdf of the $\left(\frac{m_{x}+1}{2}\right)$ th order statistics, see equation (7). 
Case 2. Even set sizes.

Let

and

$$
X_{\left(\frac{m_{x}}{2}\right) i c^{\prime}}, i=1, \ldots, \frac{m_{x}}{2} \cup X_{\left(\frac{m_{x}}{2}+1\right) i c^{\prime}} i=\frac{m_{x}}{2}+1, \ldots m_{x} ; c=1, \ldots, r_{x}
$$

$$
Y_{\left(\frac{m_{y}}{2}\right) j l^{\prime}} j=1, \ldots, \frac{m_{y}}{2} \cup Y_{\left(\frac{m_{y}}{2}+1\right) j l^{\prime}} j=\frac{m_{y}}{2}+1, \ldots m_{y} ; l=1, \ldots, r_{y},
$$

be the MRSS samples. Then the likelihood function is given by

$$
\begin{aligned}
L= & \prod_{c=1}^{r_{x}} \prod_{i=1}^{\frac{m_{x}}{2}} f_{\frac{m_{x}}{2}: m_{x}}\left(x_{\left(\frac{m_{x}}{2}\right) i c}\right) \prod_{c=1}^{r_{x}} \prod_{i=\frac{m_{x}}{2}+1}^{m_{x}} f_{\frac{m_{x}}{2}+1: m_{x}}\left(x_{\left(\frac{m_{x}}{2}+1\right) i c}\right) \\
& \cdot \prod_{l=1}^{r_{y}} \prod_{j=1}^{\frac{m_{y}}{2}} f_{\frac{m_{y}}{2}: m_{y}}\left(y_{\left(\frac{m_{y}}{2}\right) j l}\right) \prod_{l=1}^{r_{y}} \prod_{j=\frac{m_{y}}{2}+1}^{m_{y}} f_{\frac{m_{y}}{2}+1: m_{y}}\left(y_{\left(\frac{m_{y}}{2}+1\right) j l}\right) .
\end{aligned}
$$

where

$f_{\frac{m_{x}}{2}: m_{x}}\left(x_{\left(\frac{m_{x}}{2}\right)}\right)=\frac{m_{x} !}{\left(m_{x}-1\right) !\left(\frac{m_{x}}{2}-1\right) !} f\left(x_{\left(\frac{m_{x}}{2}\right)}\right)\left[F\left(x_{\left(\frac{m_{x}}{2}\right)}\right)\right]^{\frac{m_{x}}{2}-1}\left[1-F\left(x_{\left(\frac{m_{x}}{2}\right)}\right)\right]^{\frac{m_{x}}{2}}$

and

$f \frac{m_{\chi}}{2}+1: m_{x}\left(x_{\left(\frac{m_{x}}{2}+1\right)}\right)=\frac{m_{x} !}{\left(m_{x}-1\right) !\left(\frac{m_{x}}{2}\right) !} f\left(x_{\left(\frac{m_{x}}{2}+1\right)}\right)\left[F\left(x_{\left(\frac{m_{x}}{2}+1\right)}\right)\right]^{\frac{m_{x}}{2}}\left[1-F\left(x_{\left(\frac{m_{x}}{2}+1\right)}\right)\right]^{\frac{m_{x}}{2}-1}$

are the pdfs of the $\left(\frac{m_{x}}{2}\right)$ th and the $\left(\frac{m_{x}}{2}+1\right)$ th order statistics, respectively. $f_{\frac{m_{y}}{2}: m_{y}}\left(y_{\left(\frac{m_{y}}{2}\right)}\right)$ and $f_{\frac{m_{y}}{2}+1: m_{y}}\left(y_{\left(\frac{m_{y}}{2}+1\right)}\right)$ are defined similarly as shown in (14) and (15), respectively.

\section{Likelihood functions for PRSS:}

Case 1. Odd set sizes.

Let

$X_{\left(a_{x}\right) i c}, i=1, \ldots, \frac{m_{x}-1}{2} \cup X_{\left(\frac{m_{x}+1}{2}\right) i c}, i=\frac{m_{x}+1}{2} \cup X_{\left(b_{x}\right) i c}, i=\frac{m_{x}+1}{2}+1, \ldots m_{x} ; c=1, \ldots, r_{x}$ and

$Y_{\left(a_{y}\right) j l}, j=1, \ldots, \frac{m_{y}-1}{2} \cup Y_{\left(\frac{m_{y}+1}{2}\right) j l}, j=\frac{m_{y}+1}{2} \cup Y_{\left(b_{y}\right) j l}, j=\frac{m_{y}+1}{2}+1, \ldots m_{y} ; l=1, \ldots, r_{y}$, be PRSS samples. The likelihood function is

$$
\begin{aligned}
L= & \prod_{c=1}^{r_{x}} \prod_{i=1}^{\frac{m_{x}-1}{2}} f_{a_{x}: m_{x}}\left(x_{\left(a_{x}\right) i c}\right) \prod_{c=1}^{r_{x}} f_{\frac{m_{x}+1}{2}: m_{x}}\left(x_{\left(\frac{m_{x}+1}{2}\right) \frac{m_{x}+1}{2}}\right) \prod_{c=1}^{r_{x}} \prod_{i=\frac{m_{x}+1}{2}+1}^{m_{x}} f_{b_{x}: m_{x}}\left(x_{\left(b_{x}\right) i c}\right) \\
& \cdot \prod_{l=1}^{r_{y}} \prod_{j=1}^{\frac{m_{y}-1}{2}} f_{a_{y}: m_{y}}\left(y_{\left(a_{y}\right) j l}\right) \prod_{l=1}^{r_{y}} f_{\frac{m_{y}+1}{2}: m_{y}}\left(y_{\left(\frac{m_{y}+1}{2}\right) \frac{m_{y}+1}{2} l}\right) \prod_{l=1}^{r_{y}} \prod_{j=\frac{m_{y}+1}{2}+1}^{m_{y}} f_{b_{y}: m_{y}}\left(y_{\left(b_{y}\right) j l}\right)
\end{aligned}
$$


where $0<p<1, q=1-p$ and $a_{x}=\left(p\left(m_{x}+1\right)\right), b_{x}=\left(q\left(m_{x}+1\right)\right) ; a_{y}=\left(p\left(m_{y}+\right.\right.$ 1)) and $b_{y}=\left(q\left(m_{y}+1\right)\right) \cdot a_{x}, b_{x}, a_{y}$ and $b_{y}$ represent the nearest integer value. Here,

$$
\begin{aligned}
& f_{a_{x}: m_{x}}\left(x_{\left(a_{x}\right)}\right)=\frac{m_{x} !}{\left(m_{x}-1\right) !\left(a_{x}-1\right) !} f\left(x_{\left(a_{x}\right)}\right)\left[F\left(x_{\left(a_{x}\right)}\right)\right]^{a_{x}-1}\left[1-F\left(x_{\left(a_{x}\right)}\right)\right]^{m_{x}-a_{x}}, \\
& f_{b_{x}: m_{x}}\left(x_{\left(b_{x}\right)}\right)=\frac{m_{x} !}{\left(m_{x}-1\right) !\left(b_{x}-1\right) !} f\left(x_{\left(b_{x}\right)}\right)\left[F\left(x_{\left(b_{x}\right)}\right)\right]^{b_{x}-1}\left[1-F\left(x_{\left(b_{x}\right)}\right)\right]^{m_{x}-b_{x}}
\end{aligned}
$$

are the pdfs of the $\left(a_{x}\right)$ th and the $\left(b_{x}\right)$ th order statistics. Similarly, $f_{a_{y}: m_{y}}\left(y_{\left(a_{y}\right)}\right)$ and $f_{b_{y}: m_{y}}\left(y_{\left(b_{y}\right)}\right)$ are defined as in (17) and (18), respectively.

Case 2. Even set sizes.

Let

$$
X_{\left(a_{x}\right) i c}, i=1, \ldots, \frac{m_{x}}{2} \cup X_{\left(b_{x}\right) i c}, i=\frac{m_{x}}{2}+1, \ldots m_{x} ; c=1, \ldots, r_{x}
$$

and

$$
Y_{\left(a_{y}\right) j l}, j=1, \ldots, \frac{m_{y}}{2} \cup Y_{\left(b_{y}\right) j l}, j=\frac{m_{y}}{2}+1, \ldots m_{y} ; l=1, \ldots, r_{y},
$$

be PRSS samples. Then the likelihood function is shown below

$$
\begin{aligned}
L= & \prod_{c=1}^{r_{x}} \prod_{i=1}^{\frac{m_{x}}{2}} f_{a_{x}: m_{x}}\left(x_{\left(a_{x}\right) i c}\right) \prod_{c=1}^{r_{x}} \prod_{i=\frac{m_{x}}{2}+1}^{m_{x}} f_{b_{x}: m_{x}}\left(x_{\left(b_{x}\right) i c}\right) \\
& \cdot \prod_{l=1}^{r_{y}} \prod_{j=1}^{\frac{m_{y}}{2}} f_{a_{y}: m_{y}}\left(y_{\left(a_{y}\right) j l}\right) \prod_{l=1}^{r_{y}} \prod_{j=\frac{m_{y}}{2}+1}^{m_{y}} f_{b_{y}: m_{y}}\left(y_{\left(b_{y}\right) j l}\right) .
\end{aligned}
$$

\section{ML estimation based on ERSS, MRSS and PRSS:}

The solutions of the likelihood equations given for each modification and for each case are the ML estimators of the unknown parameters $p, \sigma_{1}$ and $\sigma_{2}$. However, these equations do not have explicit solutions because of the non-linear functions, $h_{1}(x)=\ln x, h_{2}(x)=x^{p}$ and $h_{3}(x)=\frac{x^{p}}{e^{x^{p} / \sigma_{1-1}}} ; h_{1}(y)=\ln y, h_{2}(y)=y^{p}$ and $h_{3}(y)=\frac{y^{p}}{e^{y^{p} / \sigma_{2}-1}}$. Therefore, we resort to iterative methods.

The ML estimators of the unknown parameters $p, \sigma_{1}$ and $\sigma_{2}$ are represented by $\hat{p}, \hat{\sigma}_{1}$ and $\hat{\sigma}_{2}$. By incorporating these estimators into (3), we obtain the ML estimators of $R$ based on ERSS, MRSS and PRSS as shown below

$$
\begin{aligned}
& \hat{R}_{M L, E R S S}=\frac{\widehat{\sigma}_{2} M L, E R S S}{\widehat{\sigma}_{1_{M L, E R S S}}+\widehat{\sigma}_{2}{ }_{M L, E R S S}}, \\
& \hat{R}_{M L, M R S S}=\frac{\widehat{\sigma}_{2_{M L, M R S S}}}{\widehat{\sigma}_{1_{M L, M R S S}+\widehat{\sigma}_{2 M L, M R S S}}}, \\
& \hat{R}_{M L, P R S S}=\frac{\widehat{\sigma}_{2_{M L, P R S S}}}{\widehat{\sigma}_{1_{M L, P R S S}}+\widehat{\sigma}_{2_{M L, P R S S}}},
\end{aligned}
$$

respectively. 


\section{MML Estimators of $R$}

In the previous section, we obtain the ML estimators of $R$ based on the modifications of RSS using iterative methods. In this section, explicit estimators of $R$ are obtained using the MML methodology for each corresponding modification. The cases of odd end even set sizes are considered separately as before.

MML methodology is applied to distributions belonging to the location-scale family. Therefore, we take the logarithm of the Weibull random variable and obtain the Extreme Value (EV) random variable which belongs to the location-scale family. In other words, if the random variable $X$ has Weibull distribution, then $U=\ln X$ has EV distribution with the following pdf and cdf

$$
f_{U}(u, \mu, \eta)=\frac{1}{\eta} e^{\left(\frac{u-\mu}{\eta}-e^{\frac{u-\mu}{\eta}}\right)},-\infty<u<\infty
$$

and

$$
F_{U}(u, \mu, \eta)=1-e^{-e^{\left(\frac{u-\mu}{\eta}\right)}},-\infty<u<\infty
$$

where $\mu \in R$ is the location parameter and $\eta \in R^{+}$is the scale parameter.

The parameters of the EV distribution can be expressed in terms of the parameters of the Weibull distribution as shown below

$$
\mu=\frac{1}{p} \ln \sigma \text { and } \eta=\frac{1}{p} \text {. }
$$

It is clear from these equalities that after obtaining the estimators of the location and the scale parameters of the EV distribution, the scale and the shape parameters of the Weibull distribution are obtained using the following inverse transformations

$$
\sigma=e^{\mu p} \text { and } p=\frac{1}{\eta}
$$

respectively.

In the context of the stress-strength model, if both the random variables $X$ and $Y$ are Weibull, then their logarithms $U=\ln X$ and $V=\ln Y$ have EV distribution which is denoted by $U \sim E V\left(\mu_{1}, \eta\right)$ and $V \sim E V\left(\mu_{2}, \eta\right)$, respectively.

\section{Estimators of the EV parameters based on ERSS:}

In this section, we derive the MML estimator of $\mu_{1}, \mu_{2}$ and $\eta$ under ERSS when the set sizes are both odd and even.

Case 1. Odd set sizes.

Let

$$
U_{(1) i c}, i=1, \ldots, \frac{m_{x}-1}{2} \cup U_{\left(\frac{m_{x}+1}{2}\right) i c}, i=\frac{m_{x}+1}{2} \cup U_{\left(m_{x}\right) i c}, i=\frac{m_{x}+1}{2}+1, \ldots m_{x} ; c=1, \ldots, r_{x}
$$

and

$$
V_{(1) j l}, j=1, \ldots, \frac{m_{y}-1}{2} \cup V_{\left(\frac{m_{y}+1}{2}\right) j l}, j=\frac{m_{y}+1}{2} \cup V_{\left(m_{y}\right) j l}, j=\frac{m_{y}+1}{2}+1, \ldots m_{y} ; l=1, \ldots, r_{y}
$$

be the ERSS samples. Here, it should be noted that the order of the observations in the ERSS sample may not be perfect. Therefore, we order them from the smallest to the largest 
to eliminate the effect of imperfect ranking. It is known that total sums are invariant to ordering, i.e., $\sum_{i=1}^{n} a_{i}=\sum_{i=1}^{n} a_{(i)}$. Similar statements can also be made for the MRSS and ERSS. We therefore do not mention it later in the paper for brevity.

The likelihood function can be written as follows

$$
\begin{gathered}
L=\frac{1}{\eta^{n+m}} \prod_{c=1}^{r_{x}} \prod_{i=1}^{\frac{m_{x}-1}{2}} f_{1: m_{x}}\left(z_{(1) i c}\right) \prod_{c=1}^{r_{x}} f_{\frac{m_{x}+1}{2}: m_{x}}\left(z_{\left.\left(\frac{m_{x}+1}{2}\right) \frac{m_{x}+1}{2} c\right)} \prod_{c=1}^{r_{x}} \prod_{i=\frac{m_{x}+1}{2}+1}^{m_{x}} f_{m_{x}: m_{x}}\left(z_{\left(m_{x}\right) i c}\right)\right. \\
. \prod_{l=1}^{r_{y} \frac{m_{y}-1}{2}} \prod_{j=1}^{r_{y}} f_{1: m_{y}}\left(w_{(1) j l}\right) \prod_{l=1}^{r_{y}} f_{\frac{m_{y}+1}{2}: m_{y}}\left(w_{\left.\left(\frac{m_{y}+1}{2}\right) \frac{m_{y}+1}{2} l\right)} \prod_{l=1}^{m_{y}} \prod_{j=\frac{m_{y}+1}{2}+1} f_{m_{y}: m_{y}}\left(w_{\left(m_{y}\right) j l}\right)\right.
\end{gathered}
$$

Here,

$$
\begin{aligned}
& z_{(1) i c}=\left(\left(u_{(1) i c}-\mu_{1}\right) / \eta\right), i=1, \ldots, \frac{m_{x}-1}{2}, c=1, \ldots, r_{x} \\
& z_{\left(\frac{m_{x}+1}{2}\right) \frac{m_{x}+1}{2} c}=\left(\left(u_{\left(\frac{m_{x}+1}{2}\right) \frac{m_{x}+1}{2} c}-\mu_{1}\right) / \eta\right), c=1, \ldots, r_{x} \text { and } \\
& z_{\left(m_{x}\right) i c}=\left(\left(u_{\left(m_{x}\right) i c}-\mu_{1}\right) / \eta\right), i=\frac{m_{x}+1}{2}+1, \ldots, m_{x}, c=1, \ldots, r_{x}
\end{aligned}
$$

are the standardized the smallest ordered statistic, median and the largest ordered statistic, respectively. Moreover, $w_{(1) j l}, w_{\left(\frac{m_{y}+1}{2}\right) \frac{m_{y}+1}{2} l}$ and $w_{\left(m_{y}\right)}$ are defined similarly as in (28).

Then, the likelihood equations are obtained as follows

$$
\begin{aligned}
& \frac{\partial \ln L}{\partial \mu_{1}}=-\frac{1}{\eta} \sum_{c=1}^{r_{x}} \sum_{i=1}^{m^{\prime}} g_{1}\left(z_{(1) i c}\right)+\frac{m_{x}-1}{\eta} \sum_{c=1}^{r_{x}} \sum_{i=1}^{m^{\prime}} g_{2}\left(z_{(1) i c}\right)-\frac{1}{\eta} \sum_{c=1}^{r_{x}} \sum_{i=m^{\prime}+2}^{m_{x}} g_{1}\left(z_{\left(m_{x}\right) i c}\right) \\
& -\frac{m_{x}-1}{\eta} \sum_{c=1}^{r_{x}} \sum_{i=m^{\prime}+2}^{m_{x}} g_{3}\left(z_{\left(m_{x}\right) i c}\right)-\frac{1}{\eta} \sum_{c=1}^{r_{x}} g_{1}\left(z_{\left(m^{\prime}+1\right) m^{\prime}+1 c}\right) \\
& +\frac{m^{\prime}}{\eta} \sum_{c=1}^{r_{x}} g_{2}\left(z_{\left(m^{\prime}+1\right) m^{\prime}+1 c}\right)-\frac{m^{\prime}}{\eta} \sum_{c=1}^{r_{x}} g_{3}\left(z_{\left(m^{\prime}+1\right) m^{\prime}+1 c}\right)=0, \\
& \frac{\partial \ln L}{\partial \mu_{2}}=-\frac{1}{\eta} \sum_{l=1}^{r_{y}} \sum_{j=1}^{m^{\prime \prime}} g_{1}\left(w_{(1) j l}\right)+\frac{m_{y}-1}{\eta} \sum_{l=1}^{r_{y}} \sum_{j=1}^{m^{\prime \prime}} g_{2}\left(w_{(1) j l}\right)-\frac{1}{\eta} \sum_{l=1}^{r_{y}} \sum_{j=m^{\prime \prime}+2}^{m_{y}} g_{1}\left(w_{\left(m_{y}\right) j l}\right) \\
& -\frac{m_{y}-1}{\eta} \sum_{l=1}^{r_{y}} \sum_{j=m^{\prime \prime}+2}^{m_{y}} g_{3}\left(w_{\left(m_{y}\right) j l}\right)-\frac{1}{\eta} \sum_{l=1}^{r_{y}} g_{1}\left(w_{\left(m^{\prime \prime}+1\right) m^{\prime \prime}+1 l}\right) \\
& +\frac{m^{\prime \prime}}{\eta} \sum_{c=1}^{r_{y}} g_{2}\left(w_{\left(m^{\prime \prime}+1\right) m^{\prime \prime}+1 l}\right)-\frac{m^{\prime \prime}}{\eta} \sum_{c=1}^{r_{y}} g_{3}\left(w_{\left(m^{\prime \prime}+1\right) m^{\prime \prime}+1 l}\right)=0, \\
& \frac{\partial \ln L}{\partial \eta}=-\frac{n+m}{\eta}-\frac{1}{\eta} \sum_{c=1}^{r_{x}} \sum_{i=1}^{m^{\prime}} g_{1}\left(z_{(1) i c}\right) \mathrm{z}_{(1) \mathrm{ic}}+\frac{m_{x}-1}{\eta} \sum_{c=1}^{r_{x}} \sum_{i=1}^{m^{\prime}} g_{2}\left(z_{(1) i c}\right) z_{(1) i c} \\
& -\frac{1}{\eta} \sum_{c=1}^{r_{x}} \sum_{i=m^{\prime}+2}^{m_{x}} g_{1}\left(z_{\left(m_{x}\right) i c}\right) z_{\left(m_{x}\right) i c}-\frac{m_{x}-1}{\eta} \sum_{c=1}^{r_{x}} \sum_{i=m^{\prime}+2}^{m_{x}} g_{3}\left(z_{\left(m_{x}\right) i c}\right) z_{\left(m_{x}\right) i c}
\end{aligned}
$$




$$
\begin{aligned}
& -\frac{1}{\eta} \sum_{l=1}^{r_{y}} \sum_{j=1}^{m^{\prime \prime}} g_{1}\left(w_{(1) j l}\right) w_{(1) j l}+\frac{m_{y}-1}{\eta} \sum_{l=1}^{r_{y}} \sum_{j=1}^{m^{\prime \prime}} g_{2}\left(w_{(1) j l}\right) w_{(1) j l} \\
& -\frac{1}{\eta} \sum_{l=1}^{r_{y}} \sum_{j=m^{\prime \prime}+2}^{m_{y}} g_{1}\left(w_{\left(m_{y}\right) j l}\right) w_{\left(m_{y}\right) j l}-\frac{m_{y}-1}{\eta} \sum_{l=1}^{r_{y}} \sum_{j=m^{\prime \prime}+2}^{m_{y}} g_{3}\left(w_{\left(m_{y}\right) j l}\right) w_{\left(m_{y}\right) j l} \\
& -\frac{1}{\eta} \sum_{c=1}^{r_{x}} g_{1}\left(z_{\left(m^{\prime}+1\right) m^{\prime}+1 c}\right) z_{\left(m^{\prime}+1\right) m^{\prime}+1 c}+\frac{m^{\prime}}{\eta} \sum_{c=1}^{r_{x}} g_{2}\left(z_{\left(m^{\prime}+1\right) m^{\prime}+1 c}\right) z_{\left(m^{\prime}+1\right) m^{\prime}+1 c} \\
& -\frac{m^{\prime}}{\eta} \sum_{c=1}^{r_{x}} g_{3}\left(z_{\left(m^{\prime}+1\right) m^{\prime}+1 c}\right) z_{\left(m^{\prime}+1\right) m^{\prime}+1 c}-\frac{1}{\eta} \sum_{l=1}^{r_{y}} g_{1}\left(w_{\left(m^{\prime \prime}+1\right) m^{\prime \prime}+1 l}\right) w_{\left(m^{\prime \prime}+1\right) m^{\prime \prime}+1 l} \\
& +\frac{m^{\prime \prime}}{\eta} \sum_{l=1}^{r_{y}} g_{2}\left(w_{\left.\left(m^{\prime \prime}+1\right) m^{\prime \prime}+1 l\right)}\right) w_{\left(m^{\prime \prime}+1\right) m^{\prime \prime}+1 l} \\
& -\frac{m^{\prime \prime}}{\eta} \sum_{l=1}^{r_{y}} g_{3}\left(w_{\left(m^{\prime \prime}+1\right) m^{\prime \prime}+1 l}\right) w_{\left(m^{\prime \prime}+1\right) m^{\prime \prime}+1 l}=0 .
\end{aligned}
$$

where $m^{\prime}=\frac{m_{x}-1}{2}$ and $m^{\prime \prime}=\frac{m_{y}-1}{2}$.

These equations do not admit explicit solutions because of the non-linear functions:

$g_{1}\left(z_{(k) i c}\right)=\frac{f^{\prime}\left(z_{(k) i c}\right)}{f\left(z_{(k) i c}\right)}, g_{2}\left(z_{(k) i c}\right)=\frac{f\left(z_{(k) i c}\right)}{1-F\left(z_{(k) i c}\right)}, g_{3}\left(z_{(k) i c}\right)=\frac{f\left(z_{(k) i c}\right)}{F\left(z_{(k) i c}\right)}, k=1, \frac{m_{x}+1}{2}, m_{x} ;$

$g_{1}\left(w_{(k) j l}\right)=\frac{f^{\prime}\left(w_{(k) j l}\right)}{f\left(w_{(k) j l}\right)}, g_{2}\left(w_{(k) j l}\right)=\frac{f\left(w_{(k) j l}\right)}{1-F\left(w_{(k) j l}\right)}, g_{3}\left(w_{(k) j l}\right)=\frac{f\left(w_{(k) j l}\right)}{F\left(w_{(k) j l}\right)}, k=1, \frac{m_{y}+1}{2}, m_{y}$.

To obtain the MML estimators, we linearize these non-linear functions as below

$$
\begin{aligned}
& g_{1}\left(z_{(k) i c}\right) \cong \alpha_{1 k}^{u}-\beta_{1 k}^{u} z_{(k) i c}, g_{2}\left(z_{(k) i c}\right) \cong \alpha_{2 k}^{u}+\beta_{2 k}^{u} z_{(k) i c}, \\
& g_{3}\left(z_{(k) i c}\right) \cong \alpha_{3 k}^{u}-\beta_{3 k}^{u} z_{(k) i c}, k=1, \frac{m_{x}+1}{2}, m_{x} ; \\
& \quad g_{1}\left(w_{(k) j l}\right) \cong \alpha_{1 k}^{v}-\beta_{1 k}^{v} w_{(k) j l}, g_{2}\left(w_{(k) j l}\right) \cong \alpha_{2 k}^{v}+\beta_{2 k}^{v} w_{(k) j l}, \\
& g_{3}\left(w_{(k) j l}\right) \cong \alpha_{3 k}^{v}-\beta_{3 k}^{v} w_{(k) j l}, k=1, \frac{m_{y}+1}{2}, m_{y},
\end{aligned}
$$

by using the first two terms of the Taylor series expansion around the expected values of the $k t h$ standardized ordered statistics, i.e., $t_{k}^{u}=E\left(z_{(k) i c}\right), k=1, \frac{m_{x}+1}{2}, m_{x}$ and $t_{k}^{v}=$ $E\left(w_{(k) j l}\right), k=1, \frac{m_{y}+1}{2}, m_{y}$. Here,

$$
\begin{aligned}
& \alpha_{1 k}^{u}=1-e^{t_{k}^{u}}+t_{k}^{u} e^{t_{k}^{u}}, \quad \beta_{1 k}^{u}=e^{t_{k}^{u}} \\
& \alpha_{2 k}^{u}=e^{t_{k}^{u}}-t_{k}^{u} e^{t_{k}^{u}}, \quad \beta_{2 k}^{u}=e^{t_{k}^{u}} \\
& \alpha_{3 k}^{u}=\frac{f\left(t_{k}^{u}\right)}{F\left(t_{k}^{u}\right)}+\beta_{3 k}^{u} t_{k}^{u}, \beta_{3 k}^{u}=\frac{\left(e^{t_{k}^{u}}\right) f\left(t_{k}^{u}\right) F\left(t_{k}^{u}\right)+f\left(t_{k}^{u}\right)^{2}}{F\left(t_{k}^{u}\right)^{2}}, \\
& t_{k}^{u}=\ln \left(-\ln \left(1-\frac{k}{m_{x}+1}\right)\right), \quad k=1, \frac{m_{x}+1}{2}, m_{x}
\end{aligned}
$$


$\left(\alpha_{1 k}^{v}, \beta_{1 k}^{v}\right),\left(\alpha_{2 k}^{v}, \beta_{2 k}^{v}\right)$ and $\left(\alpha_{3 k}^{v}, \beta_{3 k}^{v}\right)$ are exactly the same as in (34) except that $t_{k}^{u}$ are replaced by $t_{k}^{v}$. $t_{k}^{u}$ and $t_{k}^{v}$ are obtained from the following equations

$$
\begin{aligned}
& \int_{-\infty}^{t_{k}^{u}} f(z) d z=\frac{k}{m_{x}+1}, k=1, \frac{m_{x}+1}{2}, m_{x} \text { and } \\
& \int_{-\infty}^{t_{k}^{v}} f(w) d w=\frac{k}{m_{y}+1}, k=1, \frac{m_{y}+1}{2}, m_{y},
\end{aligned}
$$

respectively.

By incorporating equations (33) in equations (29)-(31), modified likelihood equations are obtained. The solutions of these equations are the following MML estimators

$$
\begin{aligned}
& \hat{\mu}_{1_{M M L, E R S S}}=K_{1}-\frac{\Delta_{1}}{m_{1}} \hat{\eta}_{M M L, E R S S}, \quad \hat{\mu}_{2_{M M L, E R S S}}=K_{2}-\frac{\Delta_{2}}{m_{2}} \hat{\eta}_{M M L, E R S S} \\
& \hat{\eta}_{M M L, E R S S}=\frac{-B+\sqrt{B^{2}+4 A C}}{2(n+m)},
\end{aligned}
$$

where

$$
\begin{aligned}
& \delta_{1}^{u}=\beta_{11}^{u}+\left(m_{x}-1\right) \beta_{21}^{u}, \quad \delta_{2}^{u}=\beta_{1 m_{x}}^{u}+\left(m_{x}-1\right) \beta_{3 m_{x}}^{u}, \\
& \delta_{3}^{u}=\left(\frac{m_{x}-1}{2}\right) \beta_{1 \frac{m_{x}+1}{2}}^{u}+\left(\frac{m_{x}-1}{2}\right) \beta_{2 \frac{m_{x}+1}{2}}^{u}+\beta_{3 \frac{m_{x}+1}{2}}^{u}, m_{1}=r_{x}\left[\frac{m_{x}-1}{2}\left(\delta_{1}^{u}+\delta_{2}^{u}\right)+\delta_{3}^{u}\right] \text {, } \\
& K_{1}=\frac{\delta_{1}^{u} \sum_{c=1}^{r_{x}} \sum_{i=1}^{\frac{m_{x}-1}{2}} u_{(1) i c}+\delta_{2}^{u} \sum_{c=1}^{r_{x}} \sum_{i=\frac{m_{x}+1}{2}+1}^{m_{x}} u_{\left(m_{x}\right) i c}+\delta_{3}^{u} \sum_{c=1}^{r_{x}} u_{\left(\frac{m_{x}+1}{2}\right) \frac{m_{x}+1}{2} c}}{m_{1}}, \\
& \Delta_{1}^{u}=\alpha_{11}^{u}-\left(m_{x}-1\right) \alpha_{21}^{u}, \quad \Delta_{2}^{u}=\alpha_{1 m_{x}}^{u}+\left(m_{x}-1\right) \alpha_{3 m_{x}}^{u}, \\
& \Delta_{3}^{u}=\left(\frac{m_{x}-1}{2}\right) \alpha_{1 \frac{m_{x}+1}{2}}^{u}-\left(\frac{m_{x}-1}{2}\right) \alpha_{2 \frac{m_{x}+1}{2}}^{u}+\alpha_{3 \frac{m_{x}+1}{2}}^{u}, \Delta_{1}=r_{x}\left[\frac{m_{x}-1}{2}\left(\Delta_{1}^{u}+\Delta_{2}^{u}\right)+\Delta_{3}^{u}\right] \text {, } \\
& \delta_{1}^{v}=\beta_{11}^{v}+\left(m_{y}-1\right) \beta_{21}^{v}, \quad \delta_{2}^{v}=\beta_{1 m_{y}}^{v}+\left(m_{y}-1\right) \beta_{3 m_{y}}^{v} \text {, } \\
& \delta_{3}^{v}=\left(\frac{m_{y}-1}{2}\right) \beta_{1 \frac{m_{y}+1}{2}}^{v}+\left(\frac{m_{y}-1}{2}\right) \beta_{2 \frac{m_{y}+1}{2}}^{v}+\beta_{3 \frac{m_{y}+1}{2}}^{v}, m_{2}=r_{y}\left[\frac{m_{y}-1}{2}\left(\delta_{1}^{v}+\delta_{2}^{v}\right)+\delta_{3}^{v}\right] \text {, } \\
& K_{2}=\frac{\delta_{1}^{v} \sum_{l=1}^{r y} \sum_{j=1}^{\frac{m_{y}-1}{2}} v_{(1) j l}+\delta_{2}^{v} \sum_{l=1}^{r_{y}} \sum_{j=\frac{m_{y}+1}{2}+1}^{m_{y}} v_{\left(m_{y}\right) j l}+\delta_{3}^{v} \sum_{l=1}^{r_{y}} v\left(\frac{m_{y}+1}{2}\right) \frac{m_{y}+1}{2} l}{m_{2}}, \\
& \Delta_{1}^{v}=\alpha_{11}^{v}-\left(m_{y}-1\right) \alpha_{21}^{v}, \quad \Delta_{2}^{v}=\alpha_{1 m_{y}}^{v}+\left(m_{y}-1\right) \alpha_{3 m_{y}}^{v}, \\
& \Delta_{3}^{v}=\left(\frac{m_{y}-1}{2}\right) \alpha_{1 \frac{m_{y}+1}{2}}^{v}-\left(\frac{m_{y}-1}{2}\right) \alpha_{2 \frac{m_{y}+1}{2}}^{v}+\alpha_{3 \frac{m_{y}+1}{2}}^{v}, \Delta_{2}=r_{y}\left[\frac{m_{y}-1}{2}\left(\Delta_{1}^{v}+\Delta_{2}^{v}\right)+\Delta_{3}^{v}\right] \text {, } \\
& A=n+m \text {, } \\
& B=\Delta_{1}^{u} \sum_{c=1}^{r_{x}} \sum_{i=1}^{\frac{m_{x}-1}{2}}\left(u_{(1) i c}-K_{1}\right)+\Delta_{2}^{u} \sum_{c=1}^{r_{x}} \sum_{i=\frac{m_{x}+1}{2}+1}^{m_{x}}\left(u_{\left(m_{x}\right) i c}-K_{1}\right)+\Delta_{3}^{u} \sum_{c=1}^{r_{x}}\left(u_{\left(\frac{m_{x}+1}{2}\right) \frac{m_{x}+1}{2} c}-K_{1}\right) \\
& +\Delta_{1}^{v} \sum_{l=1}^{r_{y}} \sum_{j=1}^{\frac{m_{y}-1}{2}}\left(v_{(1) j l}-K_{2}\right)+\Delta_{2}^{v} \sum_{l=1}^{r_{y}} \sum_{j=\frac{m_{y}+1}{2}+1}^{m_{y}}\left(v_{\left(m_{y}\right) j l}-K_{2}\right)+\Delta_{3}^{v} \sum_{l=1}^{r_{y}}\left(v_{\left(\frac{m_{y}+1}{2}\right) \frac{m_{y+1}}{2} l}-K_{2}\right), \\
& C=\delta_{1}^{u} \sum_{c=1}^{r_{x}} \sum_{i=1}^{\frac{m_{x}-1}{2}}\left(u_{(1) i c}-K_{1}\right)^{2}+\delta_{2}^{u} \sum_{c=1}^{r_{x}} \sum_{i=\frac{m_{x}+1}{2}+1}^{m_{x}}\left(u_{\left(m_{x}\right) i c}-K_{1}\right)^{2}
\end{aligned}
$$




$$
\begin{aligned}
& +\delta_{3}^{u} \sum_{c=1}^{r_{x}}\left(u_{\left(\frac{m_{x}+1}{2}\right)} \frac{m_{x}+1}{2} c-K_{1}\right)^{2}+\delta_{1}^{v} \sum_{l=1}^{r_{y}} \sum_{j=1}^{\frac{m_{y}-1}{2}}\left(v_{(1) j l}-K_{2}\right)^{2} \\
& +\delta_{2}^{v} \sum_{l=1}^{r_{y}} \sum_{j=\frac{m_{y}+1}{2}+1}^{r_{y}}\left(v_{\left(m_{y}\right) j l}-K_{2}\right)^{2}+\delta_{3}^{v} \sum_{l=1}^{r_{y}}\left(v_{\left(\frac{m_{y}+1}{2}\right) l}-K_{2}\right)^{2} .
\end{aligned}
$$

\section{Case 2. Even set sizes.}

Let

and

$$
U_{(1) i c}, i=1, \ldots, \frac{m_{x}}{2} \cup U_{\left(m_{x}\right) i c}, i=\frac{m_{x}}{2}+1, \ldots m_{x} ; c=1, \ldots, r_{x}
$$

$$
V_{(1) j l}, j=1, \ldots, \frac{m_{y}}{2} \cup V_{\left(m_{y}\right) j l}, j=\frac{m_{y}}{2}+1, \ldots m_{y} ; l=1, \ldots, r_{y}
$$

be ERSS samples. Then, the likelihood function can be written as follows

$$
\begin{gathered}
L=\frac{1}{\eta^{n+m}} \prod_{c=1}^{r_{x}} \prod_{i=1}^{\frac{m_{x}}{2}} f_{1: m_{x}}\left(z_{(1) i c}\right) \prod_{c=1}^{r_{x}} \prod_{i=\frac{m_{x}}{2}+1}^{m_{x}} f_{m_{x}: m_{x}}\left(z_{\left(m_{x}\right) i c}\right) \\
\prod_{l=1}^{r_{y}} \prod_{j=1}^{\frac{m_{y}}{2}} f_{1: m_{y}}\left(w_{(1) j l}\right) \prod_{l=1}^{r_{y}} \prod_{j=\frac{m_{y}}{2}+1}^{m_{y}} f_{m_{y}: m_{y}}\left(w_{\left(m_{y}\right) j l}\right) .
\end{gathered}
$$

where $z_{(1) i c}$ and $z_{\left(m_{x}\right) i c}$ are the standardized smallest and the largest ordered statistics. Furthermore, $w_{(1) j l}$ and $w_{\left(m_{y}\right) j l}$ are defined similarly.

Following the same steps as in Case 1, we obtain the MML estimators of $\mu_{1}, \mu_{2}$ and $\eta$ as in equation (36). We did not reproduce the details for the sake of brevity. We, therefore, just give the equalities given below. Here,

$$
\begin{aligned}
& \delta_{1}^{u}=\beta_{11}^{u}+\left(m_{x}-1\right) \beta_{21}^{u}, \quad \delta_{2}^{u}=\beta_{1 m_{x}}^{u}+\left(m_{x}-1\right) \beta_{3 m_{x}}^{u}, \\
& m_{1}=\frac{r_{x} m_{x}}{2}\left(\delta_{1}^{u}+\delta_{2}^{u}\right), K_{1}=\frac{\delta_{1}^{u} \sum_{c=1}^{r_{x}} \sum_{i=1}^{\frac{m_{x}}{2}} u_{(1) i c}+\delta_{2}^{u} \sum_{c=1}^{r_{x}} \sum_{i=\frac{m_{x}}{2}+1}^{m_{x}} u_{\left(m_{x}\right) i c}}{m_{1}}, \\
& \Delta_{1}^{u}=\alpha_{11}^{u}-\left(m_{x}-1\right) \alpha_{21}^{u}, \quad \Delta_{2}^{u}=\alpha_{1 m_{x}}^{u}+\left(m_{x}-1\right) \alpha_{3 m_{x}}^{u}, \Delta_{1}=\frac{r_{x} m_{x}}{2}\left(\Delta_{1}^{u}+\Delta_{2}^{u}\right), \\
& \delta_{1}^{v}=\beta_{11}^{v}+\left(m_{y}-1\right) \beta_{21}^{v}, \quad \delta_{2}^{v}=\beta_{1 m_{y}}^{v}+\left(m_{y}-1\right) \beta_{3 m_{y}}^{v}, \\
& m_{2}=\frac{r_{y} m_{y}}{2}\left(\delta_{1}^{v}+\delta_{2}^{v}\right), \quad K_{2}=\frac{\delta_{1}^{v} \sum_{l=1}^{r_{y}} \sum_{j=1}^{\frac{m_{y}}{2}} v_{(1) j l}+\delta_{2}^{v} \sum_{l=1}^{r y} \sum_{j=\frac{m_{y}}{2}+1}^{m_{y}} v_{\left(m_{y}\right) j l}}{m_{2}}, \\
& \Delta_{1}^{v}=\alpha_{11}^{v}-\left(m_{y}-1\right) \alpha_{21}^{v}, \quad \Delta_{2}^{v}=\alpha_{1 m_{y}}^{v}+\left(m_{y}-1\right) \alpha_{3 m_{y}}^{v}, \Delta_{2}=\frac{r_{y} m_{y}}{2}\left(\Delta_{1}^{v}+\Delta_{2}^{v}\right), \\
& A=n+m,
\end{aligned}
$$




$$
\begin{aligned}
B & =\Delta_{1}^{u} \sum_{c=1}^{r_{x}} \sum_{i=1}^{\frac{m_{x}}{2}}\left(u_{(1) i c}-K_{1}\right)+\Delta_{2}^{u} \sum_{c=1}^{r_{x}} \sum_{i=\frac{m_{x}}{2}+1}^{m_{x}}\left(u_{\left(m_{x}\right) i c}-K_{1}\right) \\
& +\Delta_{1}^{v} \sum_{l=1}^{r_{y}} \sum_{j=1}^{\frac{m_{y}}{2}}\left(v_{(1) j l}-K_{2}\right)+\Delta_{2}^{v} \sum_{l=1}^{r_{y}} \sum_{j=\frac{m_{y}}{2}+1}^{m_{y}}\left(v_{\left(m_{y}\right) j l}-K_{2}\right) \\
C & =\delta_{1}^{u} \sum_{c=1}^{r_{x}} \sum_{i=1}^{\frac{m_{x}}{2}}\left(u_{(1) i c}-K_{1}\right)^{2}+\delta_{2}^{u} \sum_{c=1}^{r_{x}} \sum_{i=\frac{m_{x}}{2}+1}^{m_{x}}\left(u_{\left(m_{x}\right) i c}-K_{1}\right)^{2} \\
& +\delta_{1}^{v} \sum_{l=1}^{r_{y}} \sum_{j=1}^{\frac{m_{y}}{2}}\left(v_{(1) j l}-K_{2}\right)^{2}+\delta_{2}^{v} \sum_{l=1}^{r_{y}} \sum_{j=\frac{m_{y}}{2}+1}^{m_{y}}\left(v_{\left(m_{y}\right) j l}-K_{2}\right)^{2} .
\end{aligned}
$$

Note: It is easy to see that the MML estimators have closed forms and are easy to compute. Moreover, the MML estimators are highly efficient even for small sample sizes. For the asymptotic properties of the MML estimators, see Vaughan and Tiku (2000) and Tiku and Akkaya (2004).

\section{Estimators of the EV parameters based on MRSS:}

We now obtain the MML estimators of $\mu_{1}, \mu_{2}$ and $\eta$ based on MRSS paying attention to the odd and even set sizes. For the sake of simplicity, we only give the MML estimators of the unknown parameters without giving the details of the derivations. Since the procedure used here is more or less the same as the procedure given in ERSS.

Case 1. Odd set sizes.

Let

$$
U_{\left(\frac{m_{x}+1}{2}\right) i c^{\prime}}, i=1, \ldots, m_{x}, c=1, \ldots, r_{x} \text { and } V_{\left(\frac{m_{y}+1}{2}\right) j l^{\prime}} j=1, \ldots, m_{y}, l=1, \ldots, r_{y}
$$

be MRSS samples. Then the likelihood function based on MRSS is given by

$$
L=\frac{1}{\eta^{n+m}} \prod_{c=1}^{r_{x}} \prod_{i=1}^{m_{x}} f_{\frac{m_{x}+1}{2}: m_{x}}\left(z_{\left(\frac{m_{x}+1}{2}\right) i c}\right) \prod_{l=1}^{r_{y}} \prod_{j=1}^{m_{y}} f_{\frac{m_{y}+1}{2}: m_{y}}\left(w_{\left(\frac{m_{y}+1}{2}\right) j l}\right) .
$$

By taking the derivatives of the log-likelihood functions with respect to the unknown parameters and equating them to zero we obtain the likelihood equations as $\frac{\partial \ln L}{\partial \ln \mu_{1}}=0$, $\frac{\partial \ln L}{\partial \ln \mu_{2}}=0$ and $\frac{\partial \ln L}{\partial \ln \eta}=0$. By linearizing non-linear functions in the likelihood equations and incorporating them into the likelihood equations, we obtain modified likelihood equations as $\frac{\partial \ln L^{*}}{\partial \ln \mu_{1}}=0, \frac{\partial \ln L^{*}}{\partial \ln \mu_{2}}=0$ and $\frac{\partial \ln L^{*}}{\partial \ln \eta}=0$. The solutions of these modified likelihood equations are the following MML estimators

where,

$$
\begin{aligned}
& \hat{\mu}_{1_{M M L, M R S S}}=K_{1}-\frac{\Delta_{1}}{m_{1}} \hat{\eta}_{M M L, M R S S}, \quad \hat{\mu}_{2_{M M L, M R S S}}=K_{2}-\frac{\Delta_{2}}{m_{2}} \hat{\eta}_{M M L, M R S S} \\
& \hat{\eta}_{M M L, M R S S}=\frac{-B+\sqrt{B^{2}+4 A C}}{2(n+m)},
\end{aligned}
$$

$\delta_{1}^{u}=\beta_{1 \frac{m_{x}+1}{2}}^{u}+\left(\frac{m_{x}-1}{2}\right) \beta_{2 \frac{m_{x}+1}{2}}^{u}+\left(\frac{m_{x}-1}{2}\right) \beta_{3 \frac{m_{x}+1}{2}}^{u}$ 


$$
\begin{aligned}
& m_{1}=r_{x} m_{x} \delta_{1}^{u}, \quad K_{1}=\frac{\delta_{1}^{u} \sum_{c=1}^{r_{x}} \sum_{i=1}^{m_{x}} u\left(\frac{m_{x}+1}{2}\right) i c}{m_{1}}, \\
& \Delta_{1}^{u}=\alpha_{1 \frac{m_{x}+1}{2}}^{u}+\left(\frac{m_{x}-1}{2}\right) \alpha_{2 \frac{m_{x}+1}{2}}^{u}-\left(\frac{m_{x}-1}{2}\right) \alpha_{3 \frac{m_{x}+1}{2}}^{u}, \Delta_{1}=r_{x} m_{x} \Delta_{1}^{u}, \\
& \delta_{1}^{v}=\beta_{1 \frac{m_{y}+1}{2}}^{v}+\left(\frac{m_{y}-1}{2}\right) \beta_{2 \frac{m_{y}+1}{2}}^{v}+\left(\frac{m_{y}-1}{2}\right) \beta_{3 \frac{m_{y}+1}{2}}^{v} \\
& m_{2}=r_{y} m_{y} \delta_{1}^{v}, K_{2}=\frac{\delta_{1}^{v} \sum_{l=1}^{r_{y}} \sum_{j=1}^{m_{y}}{ }^{m_{y}}\left(\frac{m_{y}+1}{2}\right) j l}{m_{2}}, \\
& \Delta_{1}^{v}=\alpha_{1 \frac{m_{y}+1}{2}}^{v}+\left(\frac{m_{y}-1}{2}\right) \alpha_{2 \frac{m_{y}+1}{2}}^{v}-\left(\frac{m_{y}-1}{2}\right) \alpha_{3}^{v}{ }_{\frac{m_{y}+1}{2}}, \quad \Delta_{1}=r_{y} m_{y} \Delta_{1}^{v}, \\
& A=n+m_{1}, \\
& B=\Delta_{1}^{u} \sum_{c=1}^{r_{x}} \sum_{i=1}^{m_{x}}\left(u_{\left(\frac{m_{x}+1}{2}\right) i c}-K_{1}\right)+\Delta_{1}^{v} \sum_{l=1}^{r_{y}} \sum_{j=1}^{m_{y}}\left(v_{\left(\frac{m_{y}+1}{2}\right) j l}-K_{2}\right), \\
& C=\delta_{1}^{u} \sum_{c=1}^{r_{x}} \sum_{i=1}^{m_{x}}\left(u_{\left(\frac{m_{x}+1}{2}\right) i c}-K_{1}\right)^{2}+\delta_{1}^{v} \sum_{l=1}^{r_{y}} \sum_{j=1}^{m_{y}}\left(v_{\left(\frac{m_{y}+1}{2}\right) j l}-K_{2}\right)^{2} .
\end{aligned}
$$

Case 2. Even set sizes.

Let

and

$$
U_{\left(\frac{m_{x}}{2}\right) i c^{\prime}} i=1, \ldots, \frac{m_{x}}{2} \cup U_{\left(\frac{m_{x}}{2}+1\right) i c^{\prime}} i=\frac{m_{x}}{2}+1, \ldots, m_{x} ; c=1, \ldots, r_{x}
$$

$$
V_{\left(\frac{m_{y}}{2}\right) j l}, j=1, \ldots, \frac{m_{y}}{2} \cup V_{\left(\frac{m_{y}}{2}+1\right) j l}, j=\frac{m_{y}}{2}+1, \ldots, m_{y} ; l=1, \ldots, r_{y}
$$

be MRSS samples. Then, the likelihood function is shown below

$$
\begin{aligned}
L= & \frac{1}{\eta^{n+m}} \prod_{c=1}^{r_{x}} \prod_{i=1}^{\frac{m_{x}}{2}} f_{\frac{m_{x}}{2}: m_{x}}\left(z_{\left(\frac{m_{x}}{2}\right) i c}\right) \prod_{c=1}^{r_{x}} \prod_{i=\frac{m_{x}}{2}+1}^{m_{x}} f_{\frac{m_{x}}{2}+1: m_{x}}\left(z_{\left(\frac{m_{x}}{2}+1\right) i c}\right) \\
& \cdot \prod_{l=1}^{r_{y}} \prod_{j=1}^{\frac{m_{y}}{2}} f_{\frac{m_{y}}{2}: m_{y}}\left(w\left(\frac{m_{y}}{2}\right) j l\right) \prod_{l=1}^{r_{y}} \prod_{j=\frac{m_{y}}{2}+1}^{m_{y}} f_{\frac{m_{y}}{2}+1: m_{y}}\left(w\left(\frac{m_{y}}{2}+1\right) j l\right) .
\end{aligned}
$$

Following the same MML estimation procedure shown in Case 1, we obtain the MML estimators of the parameters $\mu_{1}, \mu_{2}$ and $\eta$ as in (41), where

$$
\begin{aligned}
& \delta_{1}^{u}=\beta_{1 \frac{m_{x}}{2}}^{u}+\left(\frac{m_{x}}{2}-1\right) \beta_{2 \frac{m_{x}}{2}}^{u}+\left(\frac{m_{x}}{2}\right) \beta_{3 \frac{m_{x}}{2}}^{u}, \\
& \delta_{2}^{u}=\beta_{1 \frac{m_{x}}{2}+1}^{u}+\left(\frac{m_{x}}{2}\right) \beta_{2 \frac{m_{x}}{2}+1}^{u}+\left(\frac{m_{x}}{2}-1\right) \beta_{3 \frac{m_{x}}{2}+1}^{u}, \\
& m_{1}=r_{x} \frac{m_{x}}{2}\left(\delta_{1}^{u}+\delta_{2}^{u}\right), \quad K_{1}=\frac{\delta_{1}^{u} \sum_{c=1}^{r_{x}} \sum_{i=1}^{\frac{m_{x}}{2}} u_{\left(\frac{m_{x}}{2}\right) i c}+\delta_{2}^{u} \sum_{c=1}^{r_{x}} \sum_{i=\frac{m_{x}}{2}+1}^{m_{x}} u_{\left(\frac{m_{x}}{2}+1\right) i c}}{m_{1}} \\
& \Delta_{1}^{u}=\alpha_{1 \frac{m_{x}}{2}}^{u}+\left(\frac{m_{x}}{2}-1\right) \alpha_{2 \frac{m_{x}}{2}}^{u}-\left(\frac{m_{x}}{2}\right) \alpha_{3 \frac{m_{x}}{2}}^{u}, \\
& \Delta_{2}^{u}=\alpha_{1 \frac{m_{x}}{2}+1}^{u}+\left(\frac{m_{x}}{2}\right) \alpha_{2 \frac{m_{x}}{2}+1}^{u}-\left(\frac{m_{x}}{2}-1\right) \alpha_{3 \frac{m_{x}}{2}+1}^{u}, \Delta_{1}=r_{x} \frac{m_{x}}{2}\left(\Delta_{1}^{u}+\Delta_{2}^{u}\right),
\end{aligned}
$$




$$
\begin{aligned}
& \delta_{1}^{v}=\beta_{1 \frac{m_{y}}{2}}^{v}+\left(\frac{m_{y}}{2}-1\right) \beta_{2 \frac{m_{y}}{2}}^{v}+\left(\frac{m_{y}}{2}\right) \beta_{3 \frac{m_{y}}{2}}^{v}, \\
& \delta_{2}^{v}=\beta_{1 \frac{m_{y}}{2}+1}^{v}+\left(\frac{m_{y}}{2}\right) \beta_{2 \frac{m_{y}}{2}+1}^{v}+\left(\frac{m_{y}}{2}-1\right) \beta_{3 \frac{m_{y}}{2}+1}^{v}, \\
& m_{2}=r_{y} \frac{m_{y}}{2}\left(\delta_{1}^{v}+\delta_{2}^{v}\right), K_{2}=\frac{\delta_{1}^{v} \sum_{l=1}^{r_{y}} \sum_{j=1}^{\frac{m_{y}}{2}} v_{\left.\frac{m_{y}}{2}\right) j l}+\delta_{2}^{v} \sum_{l=1}^{r_{y}} \sum_{j=\frac{m_{y}}{2}+1}^{m_{y}}\left(\frac{m_{y}}{2}+1\right) j l}{m_{2}}, \\
& \Delta_{1}^{v}=\alpha_{1 \frac{m_{y}}{2}}^{v}+\left(\frac{m_{y}}{2}-1\right) \alpha_{2 \frac{m_{y}}{2}}^{v}-\left(\frac{m_{y}}{2}\right) \alpha_{3 \frac{m_{y}}{2}}^{v}, \\
& \Delta_{2}^{v}=\alpha_{1 \frac{m_{y}}{2}+1}^{v}+\left(\frac{m_{y}}{2}\right) \alpha_{2 \frac{m_{y}}{2}+1}^{v}-\left(\frac{m_{y}}{2}-1\right)^{v} \alpha_{3 \frac{m_{y}}{2}+1}^{v}, \quad \Delta_{2}=r_{y} \frac{m_{y}}{2}\left(\Delta_{1}^{v}+\Delta_{2}^{v}\right), \\
& A=n+m_{1}, \Delta_{c=1}^{r_{x}} \sum_{i=1}^{m_{x} / 2}\left(u_{\left(\frac{m_{x}}{2}\right) i c}-K_{1}\right)+\Delta_{2}^{u} \sum_{c=1}^{r_{x}} \sum_{i=m_{x} / 2+1}^{m_{x}} \sum_{j=1}^{m_{y} / 2}\left(u_{\left(\frac{m_{x}}{2}+1\right) i c}-K_{1}\right) j l \\
& \left.B=K_{2}\right)+\Delta_{2}^{v} \sum_{l=1}^{r_{y}} \sum_{j=m_{y} / 2+1}^{m_{y}}\left(v_{\left(\frac{m_{y}}{2}+1\right) j l}-K_{2}\right)^{2}, \\
& C=\delta_{1}^{u} \sum_{c=1}^{r_{x}} \sum_{i=1}^{m_{x} / 2}\left(u_{\left(\frac{m_{x}}{2}\right) i c}-K_{1}\right)^{2}+\delta_{2}^{u} \sum_{c=1}^{r_{x}} \sum_{i=m_{x} / 2+1}^{m_{x}}\left(u_{\left(\frac{m_{x}}{2}+1\right) i c}-K_{1}\right)^{2} \\
& +\delta_{1}^{v} \sum_{l=1}^{r_{y}} \sum_{j=1}^{m_{y} / 2}\left(v_{\left(\frac{m_{y}}{2}\right) j l}-K_{2}\right)^{2}+\delta_{2}^{v} \sum_{l=1}^{r_{y}} \sum_{j=m_{y} / 2+1}^{m_{y}}\left(v_{\left(\frac{m_{y}}{2}+1\right) j l}-K_{2}\right)^{2} .
\end{aligned}
$$

\section{MML estimator of the EV parameters based on PRSS:}

Similar to the previous sections, we obtain the MML estimator of the unknown parameters of the EV distributions based on PRSS as shown below.

Case 1. Odd set sizes.

Let

$U_{\left(a_{x}\right) i c}, i=1, \ldots, \frac{m_{x}-1}{2} \cup U_{\left(\frac{m_{x}+1}{2}\right) i c^{\prime}}, i=\frac{m_{x}+1}{2} \cup U_{\left(b_{x}\right) i c}, i=\frac{m_{x}+1}{2}+1, \ldots m_{x} ; c=1, \ldots, r_{x}$ and

$V_{\left(a_{y}\right) j l}, j=1, \ldots, \frac{m_{y}-1}{2} \cup V_{\left(\frac{m_{y}+1}{2}\right) j l}, j=\frac{m_{y}+1}{2} \cup V_{\left(b_{y}\right) j l}, j=\frac{m_{y}+1}{2}+1, \ldots m_{y} ; l=1, \ldots, r_{y}$

be PRSS samples. Here, $a_{x}$ and $b_{x} ; a_{y}$ and $b_{y}$ are define as in Section 3. The likelihood function based on PRSS is given below

$$
\begin{aligned}
L & =\frac{1}{\eta^{n+m}} \prod_{c=1}^{r_{x}} \prod_{i=1}^{\frac{m_{x}-1}{2}} f_{a_{x}: m_{x}}\left(z_{\left(a_{x}\right) i c}\right) \prod_{c=1}^{r_{x}} f_{\frac{m_{x}+1}{2}: m_{x}}\left(z_{\left(\frac{m_{x}+1}{2}\right)} \frac{m_{x}+1}{2}\right) \prod_{c=1}^{r_{x}} \prod_{i=\frac{m_{x}+1}{2}+1}^{m_{x}} f_{b_{x}: m_{x}}\left(z_{\left(b_{x}\right) i c}\right) \\
& \prod_{l=1}^{r_{y}} \frac{m_{y}-1}{2} \prod_{j=1}^{r_{y}} f_{a_{y}: m_{y}}\left(w_{\left(a_{y}\right) j l}\right) \prod_{l=1}^{r_{y}} f_{\frac{m_{y}+1}{2}: m_{y}}\left(w_{\left.\left(\frac{m_{y}+1}{2}\right) \frac{m_{y}+1}{2}\right)}\right) \prod_{l=1}^{m_{y}} \prod_{j=\frac{m_{y}+1}{2}+1}^{m_{y}} f_{b_{y}: m_{y}}\left(w_{\left(b_{y}\right) j l}\right) .
\end{aligned}
$$

As mentioned earlier, we did not give the details of the MML estimation procedure since the procedure is approximately the same as for ERSS and MRSS.

The MML estimators of $\mu_{1}, \mu_{2}$ and $\eta$ based on PRSS are denoted by $\hat{\mu}_{1_{M M L, P R S S}}, \hat{\mu}_{2_{M M L, P R S S}}$ and $\hat{\eta}_{M M L, P R S S}$ respectively: 
where,

$$
\begin{aligned}
& \hat{\mu}_{1_{M M L, P R S S}}=K_{1}-\frac{\Delta_{1}}{m_{1}} \hat{\eta}_{M M L, P R S S}, \quad \hat{\mu}_{2_{M M L, P R S S}}=K_{2}-\frac{\Delta_{2}}{m_{2}} \hat{\eta}_{M M L, P R S S} \\
& \hat{\eta}_{M M L, P R S S}=\frac{-B+\sqrt{B^{2}+4 A C}}{2(n+m)},
\end{aligned}
$$

$$
\begin{aligned}
& \delta_{1}^{u}=\beta_{1 a_{x}}^{u}+\left(a_{x}-1\right) \beta_{2 a_{x}}^{u}+\left(m_{x}-a_{x}\right) \beta_{3 a_{x}}^{u}, \\
& \delta_{2}^{u}=\beta_{1 b_{x}}^{u}+\left(b_{x}-1\right) \beta_{2 b_{x}}^{u}+\left(m_{x}-b_{x}\right) \beta_{3 b_{x}}^{u} \text {, } \\
& \delta_{3}^{u}=\beta_{1 \frac{m_{x}+1}{2}}^{u}+\left(\frac{m_{x}-1}{2}\right) \beta_{2 \frac{m_{x}+1}{2}}^{u}+\left(\frac{m_{x}-1}{2}\right) \beta_{3 \frac{m_{x}+1}{2}}^{u}, m_{1}=r_{x}\left[\frac{m_{x}-1}{2}\left(\delta_{1}^{u}+\delta_{2}^{u}\right)+\delta_{3}^{u}\right] \text {, } \\
& K_{1}=\frac{\delta_{1}^{u} \sum_{c=1}^{r_{x}} \sum_{i=1}^{\frac{m_{x}-1}{2}} u_{\left(a_{x}\right) i c}+\delta_{2}^{u} \sum_{c=1}^{r_{x}} \sum_{i=\frac{m_{x}+1}{2}+1}^{m_{x}} u_{\left(b_{x}\right) i c}+\delta_{3}^{u} \sum_{c=1}^{r_{x}} u_{\left(\frac{m_{x}+1}{2}\right) \frac{m_{x}+1}{2} c}}{m_{1}} \text {, } \\
& \Delta_{1}^{u}=\Delta_{1 a_{x}}^{u}+\left(a_{x}-1\right) \Delta_{2 a_{x}}^{u}-\left(m_{x}-a_{x}\right) \Delta_{3 a_{x}}^{u}, \\
& \Delta_{2}^{u}=\Delta_{1 b_{x}}^{u}+\left(b_{x}-1\right) \Delta_{2 b_{x}}^{u}-\left(m_{x}-b_{x}\right) \Delta_{3 b_{x}}^{u}, \\
& \Delta_{3}^{u}=\left(\frac{m_{x}-1}{2}\right) \alpha_{1 \frac{m_{x}+1}{2}}^{u}-\left(\frac{m_{x}-1}{2}\right) \alpha_{2 \frac{m_{x}+1}{2}}^{u}+\alpha_{3 \frac{m_{x}+1}{2}}^{u}, \Delta_{1}=r_{x}\left[\frac{m_{x}-1}{2}\left(\Delta_{1}^{u}+\Delta_{2}^{u}\right)+\Delta_{3}^{u}\right] \text {, } \\
& \delta_{1}^{v}=\beta_{1 a_{y}}^{v}+\left(a_{y}-1\right) \beta_{2 a_{y}}^{v}+\left(m_{y}-a_{y}\right) \beta_{3 a_{y}}^{v} \text {, } \\
& \delta_{2}^{v}=\beta_{1 b_{y}}^{v}+\left(b_{y}-1\right) \beta_{2 b_{y}}^{v}+\left(m_{y}-b_{y}\right) \beta_{3 b_{y}}^{v} \text {, } \\
& \delta_{3}^{v}=\left(\frac{m_{y}-1}{2}\right) \beta_{1 \frac{m_{y}+1}{2}}^{v}+\left(\frac{m_{y}-1}{2}\right) \beta_{2 \frac{m_{y}+1}{2}}^{v}+\beta_{3 \frac{m_{y}+1}{2}}^{v}, m_{2}=r_{y}\left[\frac{m_{y}-1}{2}\left(\delta_{1}^{v}+\delta_{2}^{v}\right)+\delta_{3}^{v}\right] \text {, } \\
& K_{2}=\frac{\delta_{1}^{v} \sum_{l=1}^{r_{y}} \sum_{j=1}^{\frac{m_{y}-1}{2}} v_{\left(a_{y}\right) j l}+\delta_{2}^{v} \sum_{l=1}^{r_{y}} \sum_{j=\frac{m_{y}+1}{2}+1}^{m_{y}} v_{\left(b_{y}\right) j l}+\delta_{3}^{v} \sum_{l=1}^{r_{y}} v_{\left(\frac{m_{y}+1}{2}\right) \frac{m_{y}+1}{2} l}}{m_{2}} \\
& \Delta_{1}^{v}=\Delta_{1 a_{y}}^{v}+\left(a_{y}-1\right) \Delta_{2 a_{y}}^{v}+\left(m_{y}-a_{y}\right) \Delta_{3 a_{y}}^{v}, \\
& \Delta_{2}^{v}=\Delta_{1 b_{y}}^{v}+\left(b_{y}-1\right) \Delta_{2 b_{y}}^{v}+\left(m_{y}-b_{y}\right) \Delta_{3 b_{y}}^{v} \\
& \Delta_{3}^{v}=\left(\frac{m_{y}-1}{2}\right) \alpha_{1 \frac{m_{y}+1}{2}}^{v}-\left(\frac{m_{y}-1}{2}\right) \alpha_{2 \frac{m_{y}+1}{2}}^{v}+\alpha_{3 \frac{m_{y}+1}{2}}^{v}, \Delta_{2}=r_{y}\left[\frac{m_{y}-1}{2}\left(\Delta_{1}^{v}+\Delta_{2}^{v}\right)+\Delta_{3}^{v}\right] \text {, } \\
& A=n+m, \\
& B=\Delta_{1}^{u} \sum_{c=1}^{r_{x}} \sum_{i=1}^{\frac{m_{x}-1}{2}}\left(u_{\left(a_{x}\right) i c}-K_{1}\right)+\Delta_{2}^{u} \sum_{c=1}^{r_{x}} \sum_{i=\frac{m_{x}+1}{2}+1}^{m_{x}}\left(u_{\left(b_{x}\right) i c}-K_{1}\right) \\
& +\Delta_{3}^{u} \sum_{c=1}^{r_{x}}\left(u_{\left(\frac{m_{x}+1}{2}\right) \frac{m_{x}+1}{2} c}-K_{1}\right)+\Delta_{1}^{v} \sum_{l=1}^{r_{y}} \sum_{j=1}^{\frac{m_{y}-1}{2}}\left(v_{\left(a_{y}\right) j l}-K_{2}\right) \\
& +\Delta_{2}^{v} \sum_{l=1}^{r_{y}} \sum_{j=\frac{m_{y}+1}{2}+1}^{m_{y}}\left(v_{\left(b_{y}\right) j l}-K_{2}\right)+\Delta_{3}^{v} \sum_{l=1}^{r_{y}}\left(v_{\left(\frac{m_{y}+1}{2}\right) \frac{m_{y}+1}{2} l}-K_{2}\right) \text {, }
\end{aligned}
$$




$$
\begin{aligned}
C & =\delta_{1}^{u} \sum_{c=1}^{r_{x}} \sum_{i=1}^{\frac{m_{x}-1}{2}}\left(u_{\left(a_{x}\right) i c}-K_{1}\right)^{2}+\delta_{2}^{u} \sum_{c=1}^{r_{x}} \sum_{i=\frac{m_{x}+1}{2}+1}^{m_{x}}\left(u_{\left(b_{x}\right) i c}-K_{1}\right)^{2} \\
& +\delta_{3}^{u} \sum_{c=1}^{r_{x}}\left(u\left(\frac{m_{x}+1}{2}\right) \frac{m_{x}+1}{2} c-K_{1}\right)^{2}+\delta_{1}^{v} \sum_{l=1}^{r_{y}} \sum_{j=1}^{\frac{m_{y}-1}{2}}\left(v_{\left(a_{y}\right) j l}-K_{2}\right)^{2} \\
& +\delta_{2}^{v} \sum_{l=1}^{r_{y}} \sum_{j=\frac{m_{y}+1}{2}+1}^{m_{y}}\left(v_{\left(b_{y}\right) j l}-K_{2}\right)^{2}+\delta_{3}^{v} \sum_{l=1}^{r_{y}}\left(v\left(\frac{m_{y}+1}{2}\right) \frac{m_{y}+1}{2} l-K_{2}\right)^{2} .
\end{aligned}
$$

\section{Case 2.}

Let

and

$$
U_{\left(a_{x}\right) i c}, i=1, \ldots, \frac{m_{x}}{2} \cup U_{\left(b_{x}\right) i c}, i=\frac{m_{x}}{2}+1, \ldots m_{x} ; c=1, \ldots, r_{x}
$$

$$
V_{\left(a_{y}\right) j l}, j=1, \ldots, \frac{m_{y}}{2} \cup V_{\left(b_{y}\right) j l}, j=\frac{m_{y}}{2}+1, \ldots m_{y} ; l=1, \ldots, r_{y}
$$

be PRSS samples. The likelihood function based on PRSS is given below

$$
\begin{aligned}
L= & \frac{1}{\eta^{n+m}} \prod_{c=1}^{r_{x}} \prod_{i=1}^{\frac{m_{x}}{2}} f_{a_{x}: m_{x}}\left(z_{\left(a_{x}\right) i c}\right) \prod_{c=1}^{r_{x}} \prod_{i=\frac{m_{x}}{2}+1}^{m_{x}} f_{b_{x}: m_{x}}\left(z_{\left(b_{x}\right) i c}\right) \\
& \cdot \prod_{l=1}^{r_{y}} \prod_{j=1}^{\frac{m_{y}}{2}} f_{a_{y}: m_{y}}\left(w_{\left(a_{y}\right) j l}\right) \prod_{l=1}^{r_{y}} \prod_{j=\frac{m_{y}}{2}+1}^{m_{y}} f_{b_{y}: m_{y}}\left(w_{\left(b_{y}\right) j l}\right) .
\end{aligned}
$$

By applying the MML procedure for the estimators of the unknown parameters, we obtain the MML estimators of $\mu_{1}, \mu_{2}$ and $\eta$ similar to (46), where

$$
\begin{aligned}
& \delta_{1}^{u}=\beta_{1 a_{x}}^{u}+\left(a_{x}-1\right) \beta_{2 a_{x}}^{u}+\left(m_{x}-a_{x}\right) \beta_{3 a_{x}}^{u}, \\
& \delta_{2}^{u}=\beta_{1 b_{x}}^{u}+\left(b_{x}-1\right) \beta_{2 b_{x}}^{u}+\left(m_{x}-b_{x}\right) \beta_{3 b_{x}}^{u}, \\
& m_{1}=r_{x} \frac{m_{x}}{2}\left(\delta_{1}^{u}+\delta_{2}^{u}\right), \quad K_{1}=\frac{\delta_{1}^{u} \sum_{c=1}^{r_{x}} \sum_{i=1}^{\frac{m_{x}}{2}} u_{\left(a_{x}\right) i c}+\delta_{2}^{u} \sum_{c=1}^{r_{x}} \sum_{i=\frac{m_{x}}{2}+1}^{m_{x}} u_{\left(b_{x}\right) i c}}{m_{1}} \\
& \Delta_{1}^{u}=\Delta_{1 a_{x}}^{u}+\left(a_{x}-1\right) \Delta_{2 a_{x}}^{u}-\left(m_{x}-a_{x}\right) \Delta_{3 a_{x}}^{u}, \\
& \Delta_{2}^{u}=\Delta_{1 b_{x}}^{u}+\left(b_{x}-1\right) \Delta_{2 b_{x}}^{u}-\left(m_{x}-b_{x}\right) \Delta_{3 b_{x}}^{u}, \Delta_{1}=r_{x} \frac{m_{x}}{2}\left(\Delta_{1}^{u}+\Delta_{2}^{u}\right), \\
& \delta_{1}^{v}=\beta_{1 a_{y}}^{v}+\left(a_{y}-1\right) \beta_{2 a_{y}}^{v}+\left(m_{y}-a_{y}\right) \beta_{3 a_{y}}^{v}, \\
& \delta_{2}^{v}=\beta_{1 b_{y}}^{v}+\left(b_{y}-1\right) \beta_{2 b_{y}}^{v}+\left(m_{y}-b_{y}\right) \beta_{3 b_{y}}^{v}, \\
& m_{2}=r_{y} \frac{m_{y}}{2}\left(\delta_{1}^{v}+\delta_{2}^{v}\right), K_{2}=\frac{\delta_{1}^{v} \sum_{l=1}^{r_{y}} \sum_{j=1}^{\frac{m_{y}}{2}} v_{\left(a_{y}\right) j l}+\delta_{2}^{v} \Sigma_{l=1}^{r_{y}} \Sigma_{j=\frac{m_{y}}{2}+1}^{m_{y}} v_{\left(b_{y}\right) j l}}{m_{2}} \\
& \Delta_{1}^{v}=\Delta_{1 a_{y}}^{v}+\left(a_{y}-1\right) \Delta_{2 a_{y}}^{v}+\left(m_{y}-a_{y}\right) \Delta_{3 a_{y}}^{v},
\end{aligned}
$$




$$
\begin{aligned}
\Delta_{2}^{v} & =\Delta_{1 b_{y}}^{v}+\left(b_{y}-1\right) \Delta_{2 b_{y}}^{v}+\left(m_{y}-b_{y}\right) \Delta_{3 b_{y}}^{v}, \Delta_{2}=r_{y} \frac{m_{y}}{2}\left(\Delta_{1}^{v}+\Delta_{2}^{v}\right), \\
A & =n+m, \\
B & =\Delta_{1}^{u} \sum_{c=1}^{r_{x}} \sum_{i=1}^{m_{x} / 2}\left(u_{\left(a_{x}\right) i c}-K_{1}\right)+\Delta_{2}^{u} \sum_{c=1}^{r_{x}} \sum_{i=m_{x} / 2+1}^{m_{x}}\left(u_{\left(b_{x}\right) i c}-K_{1}\right) \\
& +\Delta_{1}^{v} \sum_{l=1}^{r_{y}} \sum_{j=1}^{m_{y} / 2}\left(v_{\left(a_{y}\right) j l}-K_{2}\right)+\Delta_{2}^{v} \sum_{l=1}^{r_{y}} \sum_{j=m_{y} / 2+1}^{m_{y}}\left(v_{\left(b_{y}\right) j l}-K_{2}\right), \\
C & =\delta_{1}^{u} \sum_{c=1}^{r_{x}} \sum_{i=1}^{m_{x} / 2}\left(u_{\left(a_{x}\right) i c}-K_{1}\right)^{2}+\delta_{2}^{u} \sum_{c=1}^{r_{x}} \sum_{i=m_{x} / 2+1}^{m_{x}}\left(u_{\left(b_{x}\right) i c}-K_{1}\right)^{2} \\
& +\delta_{1}^{v} \sum_{l=1}^{r_{y}} \sum_{j=1}^{m_{y} / 2}\left(v_{\left(a_{y}\right) j l}-K_{2}\right)^{2}+\delta_{2}^{v} \sum_{l=1}^{r_{y}} \sum_{j=m_{y} / 2+1}^{m_{y}}\left(v_{\left(b_{y}\right) j l}-K_{2}\right)^{2} .
\end{aligned}
$$

\section{MML estimator of R based on ERSS, MRSS and PRSS:}

In this section, we obtain the MML estimators of $\mu_{1}, \mu_{2}$ and $\eta$ under ERSS, MRSS and PRSS, respectively. Using the inverse transformation given in equation (26), we derive the following MML estimators of the Weibull parameters $\sigma_{1}, \sigma_{2}$ and $p$ which are denoted by $\hat{\sigma}_{1}, \hat{\sigma}_{2}$ and $\hat{p}$, respectively;

$$
\hat{\sigma}_{1}=e^{\hat{p} \widehat{\mu}_{1}}, \quad \hat{\sigma}_{2}=e^{\hat{p} \widehat{\mu}_{2}} \text { and } \hat{p}=\frac{1}{\hat{\eta}} .
$$

By incorporating these estimators into (3), we obtain the MML estimators of the system reliability $R$ based on ERSS, MRSS and PRSS as given below

$$
\begin{aligned}
\hat{R}_{M M L, E R S S} & =\frac{\widehat{\sigma}_{2_{M M L, E R S S}}}{\hat{\sigma}_{1_{M M L, E R S S}}+\widehat{\sigma}_{2_{M M L, E R S S}}}, \\
\hat{R}_{M M L, M R S S} & =\frac{\widehat{\sigma}_{2_{M M L, M R S S}}}{\hat{\sigma}_{1_{M M L, M R S S}+\widehat{\sigma}_{2_{M M L, M R S S}}}}, \\
\hat{R}_{M M L, P R S S} & =\frac{\widehat{\sigma}_{2_{M M L, P R S S}}}{\widehat{\sigma}_{1_{M M L, P R S S}+\widehat{\sigma}_{2_{M M L, P R S S}}}},
\end{aligned}
$$

respectively.

\section{Simulation Study}

We perform an extensive Monte-Carlo simulation study in order to compare the performances of the ML and the MML estimators of $R$ based on RSS, ERSS, MRSS and PRSS with the corresponding estimators of $R$ based on SRS.

$R E$ (relative efficiency) is defined below

$$
R E=\frac{M S E\left(\hat{R}_{1}\right)}{M S E\left(\hat{R}_{2}\right)}
$$

where $\hat{R}_{1}$ and $\hat{R}_{2}$ are two different estimators of $R$ and $\operatorname{MSE}(\hat{R})=E(\hat{R}-R)^{2}$. It is known that, if the value of $R E$ in (54) is greater than 1 this means that $\hat{R}_{2}$ is more efficient than $\hat{R}_{1}$ and vice versa. In the comparisons, we use the following $R E \mathrm{~s}$

$$
\begin{aligned}
& R E_{1}=\frac{M S E\left(\hat{R}_{M L, S R S}\right)}{M S E\left(\hat{R}_{M L, R S S}\right)}, \quad R E_{2}=\frac{M S E\left(\hat{R}_{M L, S R S}\right)}{M S E\left(\hat{R}_{M L, E R S S}\right)}, \quad R E_{3}=\frac{M S E\left(\hat{R}_{M L, S R S}\right)}{M S E\left(\hat{R}_{M L, M R S S}\right)}, \quad R E_{4}=\frac{M S E\left(\hat{R}_{M L, S R S}\right)}{M S E\left(\hat{R}_{M L, P R S S}\right)}, \\
& R E_{5}=\frac{M S E\left(\hat{R}_{M M L, S R S}\right)}{M S E\left(\hat{R}_{M M L, R S S}\right)}, R E_{6}=\frac{M S E\left(\hat{R}_{M M L, S R S}\right)}{M S E\left(\hat{R}_{M M L, E R S S}\right)}, R E_{7}=\frac{M S E\left(\hat{R}_{M M L, S R S}\right)}{M S E\left(\hat{R}_{M M L, M R S S}\right)}, R E_{8}=\frac{M S E\left(\hat{R}_{M M L, S R S}\right)}{M S E\left(\hat{R}_{M M L, P R S S}\right)},
\end{aligned}
$$


$R E_{9}=\frac{M S E\left(\hat{R}_{M M L, R S S}\right)}{M S E\left(\hat{R}_{M L, R S S}\right)}, R E_{10}=\frac{M S E\left(\hat{R}_{M M L, E R S S}\right)}{M S E\left(\hat{R}_{M L, E R S S}\right)}, R E_{11}=\frac{M S E\left(\hat{R}_{M M L, M R S S}\right)}{M S E\left(\hat{R}_{M L, M R S S}\right)}, R E_{12}=\frac{M S E\left(\hat{R}_{M M L, P R S S}\right)}{M S E\left(\hat{R}_{M L, P R S S}\right)}$.

It is clear that $R E_{1}, R E_{2}, R E_{3}$ and $R E_{4}$ compare the MSEs of the ML estimators of $R$ based on RSS and its modifications to the MSE of the corresponding estimator of $R$ based on SRS. Similarly, $R E_{5}, R E_{6}, R E_{7}$ and $R E_{8}$ compare the MSEs of the MML estimators of $R$ based on RSS, ERSS, MRSS and PRSS to the MSE of the MML estimator of $R$ based on SRS. In addition, $R E_{9}, R E_{10}, R E_{11}$ and $R E_{12}$ compare the ML and the MML estimators of $R$ with respect to RSS, ERSS, MRSS and PRSS.

Monte-Carlo simulations are applied for the different set sizes $\left(m_{x}, m_{y}\right)$ and different number of cycles $\left(r_{x}, r_{y}\right)$, i.e.,

$$
\begin{aligned}
& r_{x}=r_{y}=1 \Longrightarrow\left(m_{x}, m_{y}\right)=(5,5),(5,10),(15,15),(15,20),(20,20), \\
& r_{x}=r_{y}=5 \Longrightarrow\left(m_{x}, m_{y}\right)=(2,2),(2,3),(2,4),(3,4),(4,4),
\end{aligned}
$$

and

$$
r_{x}=r_{y}=10 \Rightarrow\left(m_{x}, m_{y}\right)=(2,2),(3,3),(4,4)
$$

Sample sizes corresponding to $X$ and $Y$ are determined by $n=m_{x} r_{x}$ and $m=m_{y} r_{y}$. It is clear that when $r_{x}=r_{y}=1$, samples sizes reduce to the set sizes.

In the simulation study, we present the $R E$ s for the parameter values $\sigma_{1}=1, \sigma_{2}=1,2,3$ and $p=1.5,3,6$ consistent with other studies given in the literature. All the simulations are conducted using Matlab R2013a for $\llbracket 100,000 / \min (n, m) \rrbracket$ Monte-Carlo runs. Here, 【. \ represents the greatest integer value. Simulation results are reported in Table 1.

Note that the bias values are not reproduced in Table 1 , since $\hat{R}$ has negligible bias for all estimators, sampling methods and parameter values.

It is clear that, RSS and its modifications become equivalent when the set sizes $\left(m_{x}, m_{y}\right)=(2,2)$. Similarly, ERSS and PRSS samples reduce to RSS when the set sizes $\left(m_{x}, m_{y}\right)=(2,3)$ or $(3,3)$. 
Table 1: REs of the estimators based on RSS, ERSS, MRSS and PRSS

\begin{tabular}{|c|c|c|c|c|c|c|c|c|c|c|c|c|c|}
\hline \multicolumn{14}{|c|}{$p=1.5$} \\
\hline \multicolumn{14}{|c|}{ Efficiency } \\
\hline$r_{x}=r_{y}$ & $\left(m_{x}, m_{y}\right)$ & $R E_{1}$ & $R E_{2}$ & $R E_{3}$ & $R E_{4}$ & $R E_{5}$ & $R E_{6}$ & $R E_{7}$ & $R E_{8}$ & $R E_{9}$ & $R E_{10}$ & $R E_{11}$ & $R E_{12}$ \\
\hline \multicolumn{14}{|c|}{$\sigma_{1}=1, \sigma_{2}=1$} \\
\hline \multirow{5}{*}{1} & $(5,5)$ & 2.87 & 2.85 & 2.63 & 2.78 & 2.69 & 3.08 & 2.43 & 2.41 & 1.01 & 0.88 & 1.03 & 1.09 \\
\hline & $(5,10)$ & 3.42 & 3.09 & 3.31 & 3.56 & 3.05 & 3.53 & 2.67 & 3.42 & 1.09 & 0.86 & 1.21 & 1.02 \\
\hline & $(15,15)$ & 7.23 & 4.92 & 7.58 & 7.67 & 6.83 & 6.02 & 7.32 & 7.51 & 1.04 & 0.80 & 1.02 & 1.00 \\
\hline & $(15,20)$ & 7.92 & 5.08 & 8.47 & 8.78 & 7.38 & 6.07 & 7.46 & 8.75 & 1.06 & 0.82 & 1.12 & 0.99 \\
\hline & $(20,20)$ & 9.50 & 5.51 & 9.90 & 10.4 & 9.04 & 6.70 & 8.57 & 10.6 & 1.04 & 0.81 & 1.14 & 0.97 \\
\hline \multirow{5}{*}{5} & $(2,2)$ & 1.50 & 1.50 & 1.50 & 1.50 & 1.34 & 1.34 & 1.34 & 1.34 & 1.09 & 1.09 & 1.09 & 1.09 \\
\hline & $(2,3)$ & 1.61 & 1.61 & 1.59 & 1.61 & 1.34 & 1.34 & 1.35 & 1.34 & 1.18 & 1.18 & 1.15 & 1.18 \\
\hline & $(2,4)$ & 1.60 & 1.61 & 1.59 & 1.67 & 1.15 & 1.46 & 1.10 & 1.13 & 1.38 & 1.10 & 1.43 & 1.46 \\
\hline & $(3,4)$ & 1.94 & 1.89 & 2.00 & 2.02 & 1.75 & 1.97 & 1.54 & 1.76 & 1.09 & 0.95 & 1.29 & 1.13 \\
\hline & $(4,4)$ & 2.18 & 2.06 & 2.26 & 2.54 & 2.09 & 2.30 & 1.95 & 2.44 & 1.03 & 0.88 & 1.14 & 1.03 \\
\hline \multirow{3}{*}{10} & $(2,2)$ & 1.39 & 1.39 & 1.39 & 1.39 & 1.26 & 1.26 & 1.26 & 1.26 & 1.08 & 1.08 & 1.08 & 1.08 \\
\hline & $(3,3)$ & 1.91 & 1.91 & 1.96 & 1.91 & 1.79 & 1.79 & 1.70 & 1.79 & 1.06 & 1.06 & 1.14 & 1.06 \\
\hline & $(4,4)$ & 2.26 & 2.13 & 2.28 & 2.53 & 2.15 & 2.40 & 1.96 & 2.42 & 1.05 & 0.89 & 1.16 & 1.04 \\
\hline \multicolumn{14}{|c|}{$\sigma_{1}=1, \sigma_{2}=2$} \\
\hline \multirow{5}{*}{1} & $(5,5)$ & 2.72 & 2.73 & 2.44 & 2.65 & 2.52 & 2.85 & 2.26 & 2.29 & 1.03 & 0.92 & 1.03 & 1.11 \\
\hline & $(5,10)$ & 3.23 & 2.81 & 2.89 & 3.18 & 2.88 & 2.89 & 2.22 & 3.04 & 1.08 & 0.94 & 1.26 & 1.01 \\
\hline & $(15,15)$ & 6.87 & 4.95 & 5.33 & 6.85 & 6.27 & 4.64 & 5.20 & 6.53 & 1.08 & 1.05 & 1.01 & 1.03 \\
\hline & $(15,20)$ & 7.62 & 4.85 & 5.80 & 7.41 & 6.91 & 4.07 & 4.65 & 7.37 & 1.09 & 1.18 & 1.23 & 0.99 \\
\hline & $(20,20)$ & 8.26 & 5.26 & 6.20 & 8.58 & 7.50 & 4.38 & 4.64 & 8.57 & 1.09 & 1.19 & 1.33 & 0.99 \\
\hline \multirow{5}{*}{5} & $(2,2)$ & 1.35 & 1.35 & 1.35 & 1.35 & 1.19 & 1.19 & 1.19 & 1.19 & 1.11 & 1.11 & 1.11 & 1.11 \\
\hline & $(2,3)$ & 1.55 & 1.55 & 1.56 & 1.55 & 1.35 & 1.35 & 1.38 & 1.35 & 1.13 & 1.13 & 1.11 & 1.13 \\
\hline & $(2,4)$ & 1.63 & 1.58 & 1.66 & 1.63 & 1.29 & 1.56 & 1.22 & 1.25 & 1.24 & 1.00 & 1.33 & 1.28 \\
\hline & $(3,4)$ & 1.91 & 1.80 & 1.93 & 1.90 & 1.74 & 1.64 & 1.47 & 1.72 & 1.08 & 1.09 & 1.29 & 1.09 \\
\hline & $(4,4)$ & 2.14 & 2.04 & 2.14 & 2.41 & 1.96 & 1.90 & 1.82 & 2.27 & 1.09 & 1.07 & 1.17 & 1.05 \\
\hline \multirow{3}{*}{10} & $(2,2)$ & 1.37 & 1.37 & 1.37 & 1.37 & 1.16 & 1.16 & 1.16 & 1.16 & 1.16 & 1.16 & 1.16 & 1.16 \\
\hline & $(3,3)$ & 1.73 & 1.73 & 1.79 & 1.73 & 1.52 & 1.52 & 1.51 & 1.52 & 1.14 & 1.14 & 1.19 & 1.14 \\
\hline & $(4,4)$ & 2.25 & 2.13 & 2.32 & 2.55 & 2.02 & 1.67 & 1.94 & 2.37 & 1.11 & 1.27 & 1.19 & 1.07 \\
\hline \multicolumn{14}{|c|}{$\sigma_{1}=1, \sigma_{2}=3$} \\
\hline \multirow{5}{*}{1} & $(5,5)$ & 2.64 & 2.69 & 2.25 & 2.48 & 2.42 & 2.65 & 2.11 & 2.16 & 1.06 & 0.99 & 1.04 & 1.12 \\
\hline & $(5,10)$ & 3.14 & 2.78 & 2.59 & 3.04 & 2.77 & 2.46 & 1.99 & 2.85 & 1.09 & 1.08 & 1.25 & 1.02 \\
\hline & $(15,15)$ & 5.75 & 4.56 & 3.83 & 5.39 & 5.12 & 3.30 & 3.76 & 5.09 & 1.12 & 1.37 & 1.01 & 1.05 \\
\hline & $(15,20)$ & 6.67 & 4.63 & 4.12 & 6.36 & 5.81 & 2.78 & 3.23 & 6.24 & 1.13 & 1.64 & 1.26 & 1.00 \\
\hline & $(20,20)$ & 7.55 & 5.26 & 4.37 & 7.56 & 6.72 & 3.02 & 3.08 & 7.42 & 1.12 & 1.73 & 1.41 & 1.01 \\
\hline \multirow{5}{*}{5} & $(2,2)$ & 1.37 & 1.37 & 1.37 & 1.37 & 1.13 & 1.13 & 1.13 & 1.13 & 1.19 & 1.19 & 1.19 & 1.19 \\
\hline & $(2,3)$ & 1.52 & 1.52 & 1.56 & 1.52 & 1.32 & 1.32 & 1.39 & 1.32 & 1.13 & 1.13 & 1.10 & 1.13 \\
\hline & $(2,4)$ & 1.58 & 1.52 & 1.62 & 1.55 & 1.32 & 1.42 & 1.26 & 1.29 & 1.18 & 1.06 & 1.26 & 1.19 \\
\hline & $(3,4)$ & 1.99 & 1.86 & 1.86 & 1.93 & 1.76 & 1.49 & 1.45 & 1.74 & 1.11 & 1.23 & 1.26 & 1.09 \\
\hline & $(4,4)$ & 2.16 & 2.15 & 2.04 & 2.38 & 1.81 & 1.59 & 1.68 & 2.13 & 1.18 & 1.35 & 1.21 & 1.11 \\
\hline \multirow{3}{*}{10} & $(2,2)$ & 1.42 & 1.42 & 1.42 & 1.42 & 1.11 & 1.11 & 1.11 & 1.11 & 1.27 & 1.27 & 1.27 & 1.27 \\
\hline & $(3,3)$ & 1.60 & 1.60 & 1.63 & 1.60 & 1.28 & 1.28 & 1.37 & 1.28 & 1.25 & 1.25 & 1.18 & 1.25 \\
\hline & $(4,4)$ & 2.14 & 2.08 & 2.11 & 2.42 & 1.75 & 1.16 & 1.65 & 2.08 & 1.21 & 1.79 & 1.27 & 1.16 \\
\hline
\end{tabular}


Table 1. (continued),

\begin{tabular}{|c|c|c|c|c|c|c|c|c|c|c|c|c|c|}
\hline \multicolumn{14}{|c|}{$p=3$} \\
\hline \multicolumn{14}{|c|}{ Efficiency } \\
\hline$r_{x}=r_{y}$ & $\left(m_{x}, m_{y}\right)$ & $R E_{1}$ & $R E_{2}$ & $R E_{3}$ & $R E_{4}$ & $R E_{5}$ & $R E_{6}$ & $R E_{7}$ & $R E_{8}$ & $R E_{9}$ & $R E_{10}$ & $R E_{11}$ & $R E_{12}$ \\
\hline \multicolumn{14}{|c|}{$\sigma_{1}=1, \sigma_{2}=1$} \\
\hline \multirow{5}{*}{1} & $(5,5)$ & 2.83 & 2.82 & 2.62 & 2.80 & 2.66 & 3.07 & 2.42 & 2.43 & 1.01 & 0.87 & 1.02 & 1.09 \\
\hline & $(5,10)$ & 3.43 & 3.15 & 3.23 & 3.55 & 3.09 & 3.59 & 2.61 & 3.39 & 1.08 & 0.86 & 1.21 & 1.03 \\
\hline & $(15,15)$ & 7.60 & 5.14 & 7.94 & 8.17 & 7.15 & 6.22 & 7.66 & 7.92 & 1.04 & 0.81 & 1.02 & 1.01 \\
\hline & $(15,20)$ & 7.87 & 5.19 & 8.40 & 8.66 & 7.38 & 6.19 & 7.39 & 8.63 & 1.05 & 0.83 & 1.12 & 0.99 \\
\hline & $(20,20)$ & 9.35 & 5.60 & 10.01 & 10.31 & 8.87 & 6.78 & 8.62 & 10.46 & 1.04 & 0.82 & 1.15 & 0.98 \\
\hline \multirow{5}{*}{5} & $(2,2)$ & 1.40 & 1.40 & 1.40 & 1.40 & 1.25 & 1.25 & 1.25 & 1.25 & 1.09 & 1.09 & 1.09 & 1.09 \\
\hline & $(2,3)$ & 1.61 & 1.61 & 1.58 & 1.61 & 1.32 & 1.32 & 1.33 & 1.32 & 1.20 & 1.20 & 1.17 & 1.20 \\
\hline & $(2,4)$ & 1.61 & 1.59 & 1.58 & 1.64 & 1.14 & 1.43 & 1.09 & 1.13 & 1.40 & 1.11 & 1.44 & 1.45 \\
\hline & $(3,4)$ & 1.95 & 1.93 & 2.07 & 2.06 & 1.78 & 2.04 & 1.58 & 1.81 & 1.08 & 0.94 & 1.30 & 1.13 \\
\hline & $(4,4)$ & 2.21 & 2.10 & 2.32 & 2.54 & 2.13 & 2.40 & 1.99 & 2.44 & 1.03 & 0.87 & 1.15 & 1.03 \\
\hline \multirow{3}{*}{10} & $(2,2)$ & 1.36 & 1.36 & 1.36 & 1.36 & 1.21 & 1.21 & 1.21 & 1.21 & 1.12 & 1.12 & 1.12 & 1.12 \\
\hline & $(3,3)$ & 1.78 & 1.78 & 1.92 & 1.78 & 1.69 & 1.69 & 1.66 & 1.69 & 1.05 & 1.05 & 1.15 & 1.05 \\
\hline & $(4,4)$ & 2.07 & 1.96 & 2.17 & 2.41 & 2.00 & 2.21 & 1.89 & 2.31 & 1.03 & 0.89 & 1.14 & 1.04 \\
\hline \multicolumn{14}{|c|}{$\sigma_{1}=1, \sigma_{2}=2$} \\
\hline \multirow{5}{*}{1} & $(5,5)$ & 2.72 & 2.70 & 2.35 & 2.60 & 2.53 & 2.83 & 2.18 & 2.25 & 1.03 & 0.91 & 1.03 & 1.10 \\
\hline & $(5,10)$ & 3.18 & 2.86 & 2.83 & 3.17 & 2.82 & 2.93 & 2.16 & 3.02 & 1.09 & 0.94 & 1.26 & 1.01 \\
\hline & $(15,15)$ & 6.58 & 4.93 & 5.14 & 6.65 & 6.08 & 4.59 & 5.03 & 6.41 & 1.07 & 1.06 & 1.01 & 1.02 \\
\hline & $(15,20)$ & 7.20 & 4.91 & 5.70 & 7.15 & 6.52 & 4.04 & 4.56 & 7.12 & 1.09 & 1.20 & 1.23 & 0.99 \\
\hline & $(20,20)$ & 8.57 & 5.31 & 6.36 & 8.99 & 7.89 & 4.43 & 4.71 & 9.04 & 1.08 & 1.19 & 1.34 & 0.99 \\
\hline \multirow{5}{*}{5} & $(2,2)$ & 1.40 & 1.40 & 1.40 & 1.40 & 1.21 & 1.21 & 1.21 & 1.21 & 1.13 & 1.13 & 1.13 & 1.13 \\
\hline & $(2,3)$ & 1.59 & 1.59 & 1.61 & 1.59 & 1.39 & 1.39 & 1.44 & 1.39 & 1.11 & 1.11 & 1.09 & 1.11 \\
\hline & $(2,4)$ & 1.64 & 1.61 & 1.68 & 1.64 & 1.29 & 1.56 & 1.24 & 1.27 & 1.24 & 1.01 & 1.33 & 1.27 \\
\hline & $(3,4)$ & 1.99 & 1.89 & 1.96 & 1.96 & 1.80 & 1.75 & 1.49 & 1.77 & 1.09 & 1.07 & 1.30 & 1.09 \\
\hline & $(4,4)$ & 2.14 & 2.06 & 2.16 & 2.49 & 1.98 & 1.93 & 1.79 & 2.36 & 1.08 & 1.06 & 1.19 & 1.05 \\
\hline \multirow{3}{*}{10} & $(2,2)$ & 1.36 & 1.36 & 1.36 & 1.36 & 1.16 & 1.16 & 1.16 & 1.16 & 1.16 & 1.16 & 1.16 & 1.16 \\
\hline & $(3,3)$ & 1.79 & 1.79 & 1.85 & 1.79 & 1.63 & 1.63 & 1.60 & 1.63 & 1.09 & 1.09 & 1.16 & 1.09 \\
\hline & $(4,4)$ & 2.16 & 2.05 & 2.14 & 2.40 & 1.89 & 1.61 & 1.76 & 2.20 & 1.14 & 1.27 & 1.21 & 1.09 \\
\hline \multicolumn{14}{|c|}{$\sigma_{1}=1, \sigma_{2}=3$} \\
\hline \multirow{5}{*}{1} & $(5,5)$ & 2.60 & 2.60 & 2.23 & 2.47 & 2.39 & 2.59 & 2.10 & 2.14 & 1.06 & 0.98 & 1.04 & 1.12 \\
\hline & $(5,10)$ & 3.13 & 2.76 & 2.58 & 3.07 & 2.75 & 2.43 & 1.98 & 2.86 & 1.09 & 1.09 & 1.25 & 1.03 \\
\hline & $(15,15)$ & 6.04 & 4.73 & 3.84 & 5.60 & 5.44 & 3.38 & 3.76 & 5.37 & 1.10 & 1.39 & 1.01 & 1.04 \\
\hline & $(15,20)$ & 6.69 & 4.78 & 4.00 & 6.31 & 5.87 & 2.86 & 3.16 & 6.14 & 1.12 & 1.64 & 1.24 & 1.01 \\
\hline & $(20,20)$ & 7.24 & 4.95 & 4.23 & 7.37 & 6.50 & 2.80 & 3.03 & 7.20 & 1.11 & 1.76 & 1.39 & 1.02 \\
\hline \multirow{5}{*}{5} & $(2,2)$ & 1.40 & 1.40 & 1.40 & 1.40 & 1.15 & 1.15 & 1.15 & 1.15 & 1.20 & 1.20 & 1.20 & 1.20 \\
\hline & $(2,3)$ & 1.60 & 1.60 & 1.65 & 1.60 & 1.39 & 1.39 & 1.47 & 1.40 & 1.13 & 1.13 & 1.11 & 1.12 \\
\hline & $(2,4)$ & 1.65 & 1.59 & 1.69 & 1.63 & 1.35 & 1.47 & 1.29 & 1.33 & 1.20 & 1.05 & 1.28 & 1.20 \\
\hline & $(3,4)$ & 1.90 & 1.78 & 1.79 & 1.84 & 1.67 & 1.40 & 1.41 & 1.67 & 1.12 & 1.26 & 1.26 & 1.09 \\
\hline & $(4,4)$ & 2.10 & 2.05 & 2.01 & 2.29 & 1.82 & 1.56 & 1.65 & 2.07 & 1.15 & 1.30 & 1.21 & 1.10 \\
\hline \multirow{3}{*}{10} & $(2,2)$ & 1.37 & 1.37 & 1.37 & 1.37 & 1.10 & 1.10 & 1.10 & 1.10 & 1.24 & 1.24 & 1.24 & 1.24 \\
\hline & $(3,3)$ & 1.57 & 1.57 & 1.62 & 1.57 & 1.31 & 1.31 & 1.32 & 1.31 & 1.20 & 1.20 & 1.22 & 1.20 \\
\hline & $(4,4)$ & 2.28 & 2.26 & 2.11 & 2.52 & 1.81 & 1.24 & 1.68 & 2.17 & 1.26 & 1.82 & 1.25 & 1.16 \\
\hline
\end{tabular}


Table 1. (continued).

\begin{tabular}{|c|c|c|c|c|c|c|c|c|c|c|c|c|c|}
\hline \multicolumn{14}{|c|}{$p=6$} \\
\hline \multicolumn{14}{|c|}{ Efficiency } \\
\hline$r_{x}=r_{y}$ & $\left(m_{x}, m_{y}\right)$ & $R E_{1}$ & $R E_{2}$ & $R E_{3}$ & $R E_{4}$ & $R E_{5}$ & $R E_{6}$ & $R E_{7}$ & $R E_{8}$ & $R E_{9}$ & $R E_{10}$ & $R E_{11}$ & $R E_{12}$ \\
\hline \multicolumn{14}{|c|}{$\sigma_{1}=1, \sigma_{2}=1$} \\
\hline \multirow{5}{*}{1} & $(5,5)$ & 2.83 & 2.82 & 2.62 & 2.80 & 2.66 & 3.07 & 2.42 & 2.43 & 1.01 & 0.87 & 1.02 & 1.09 \\
\hline & $(5,10)$ & 3.43 & 3.15 & 3.23 & 3.55 & 3.09 & 3.59 & 2.61 & 3.39 & 1.08 & 0.86 & 1.21 & 1.03 \\
\hline & $(15,15)$ & 7.60 & 5.14 & 7.94 & 8.17 & 7.15 & 6.22 & 7.66 & 7.92 & 1.04 & 0.81 & 1.02 & 1.01 \\
\hline & $(15,20)$ & 7.81 & 4.96 & 8.47 & 8.45 & 7.37 & 5.94 & 7.48 & 8.48 & 1.05 & 0.82 & 1.12 & 0.99 \\
\hline & $(20,20)$ & 9.26 & 5.29 & 9.85 & 10.3 & 8.82 & 6.45 & 8.58 & 10.53 & 1.04 & 0.81 & 1.14 & 0.97 \\
\hline \multirow{5}{*}{5} & $(2,2)$ & 1.41 & 1.41 & 1.41 & 1.41 & 1.27 & 1.27 & 1.27 & 1.27 & 1.08 & 1.08 & 1.08 & 1.08 \\
\hline & $(2,3)$ & 1.62 & 1.61 & 1.60 & 1.61 & 1.34 & 1.34 & 1.36 & 1.34 & 1.18 & 1.18 & 1.16 & 1.18 \\
\hline & $(2,4)$ & 1.66 & 1.63 & 1.63 & 1.68 & 1.20 & 1.49 & 1.14 & 1.16 & 1.37 & 1.09 & 1.43 & 1.44 \\
\hline & $(3,4)$ & 1.94 & 1.89 & 2.04 & 2.04 & 1.75 & 1.97 & 1.57 & 1.78 & 1.10 & 0.95 & 1.29 & 1.14 \\
\hline & $(4,4)$ & 2.27 & 2.14 & 2.41 & 2.63 & 2.16 & 2.38 & 2.05 & 2.51 & 1.04 & 0.89 & 1.17 & 1.04 \\
\hline \multirow{3}{*}{10} & $(2,2)$ & 1.40 & 1.40 & 1.40 & 1.40 & 1.26 & 1.26 & 1.26 & 1.26 & 1.10 & 1.10 & 1.10 & 1.10 \\
\hline & $(3,3)$ & 1.86 & 1.86 & 1.86 & 1.86 & 1.71 & 1.71 & 1.62 & 1.71 & 1.08 & 1.08 & 1.15 & 1.08 \\
\hline & $(4,4)$ & 2.10 & 2.06 & 2.17 & 2.55 & 1.99 & 2.29 & 1.85 & 2.38 & 1.05 & 0.90 & 1.17 & 1.07 \\
\hline \multicolumn{14}{|c|}{$\sigma_{1}=1, \sigma_{2}=2$} \\
\hline \multirow{5}{*}{1} & $(5,5)$ & 2.79 & 2.78 & 2.47 & 2.69 & 2.56 & 2.91 & 2.30 & 2.31 & 1.04 & 0.91 & 1.03 & 1.11 \\
\hline & $(5,10)$ & 3.24 & 2.85 & 2.90 & 3.22 & 2.89 & 2.95 & 2.23 & 3.07 & 1.08 & 0.93 & 1.26 & 1.01 \\
\hline & $(15,15)$ & 6.49 & 4.86 & 5.22 & 6.61 & 5.99 & 4.71 & 5.07 & 6.35 & 1.07 & 1.02 & 1.02 & 1.03 \\
\hline & $(15,20)$ & 7.21 & 4.64 & 5.69 & 7.23 & 6.53 & 3.82 & 4.53 & 7.22 & 1.09 & 1.20 & 1.24 & 0.99 \\
\hline & $(20,20)$ & 8.48 & 5.23 & 6.24 & 8.87 & 7.82 & 4.35 & 4.72 & 8.90 & 1.08 & 1.19 & 1.31 & 0.99 \\
\hline \multirow{5}{*}{5} & $(2,2)$ & 1.42 & 1.42 & 1.42 & 1.42 & 1.21 & 1.21 & 1.21 & 1.21 & 1.14 & 1.14 & 1.14 & 1.14 \\
\hline & $(2,3)$ & 1.55 & 1.55 & 1.59 & 1.55 & 1.35 & 1.35 & 1.40 & 1.35 & 1.12 & 1.12 & 1.11 & 1.12 \\
\hline & $(2,4)$ & 1.63 & 1.58 & 1.66 & 1.63 & 1.29 & 1.56 & 1.22 & 1.25 & 1.24 & 1.00 & 1.33 & 1.28 \\
\hline & $(3,4)$ & 1.91 & 1.80 & 1.93 & 1.90 & 1.74 & 1.64 & 1.47 & 1.72 & 1.08 & 1.09 & 1.29 & 1.09 \\
\hline & $(4,4)$ & 2.14 & 2.04 & 2.14 & 2.41 & 1.96 & 1.90 & 1.82 & 2.27 & 1.09 & 1.07 & 1.17 & 1.05 \\
\hline \multirow{3}{*}{10} & $(2,2)$ & 1.37 & 1.37 & 1.37 & 1.37 & 1.16 & 1.16 & 1.16 & 1.16 & 1.16 & 1.16 & 1.16 & 1.16 \\
\hline & $(3,3)$ & 1.73 & 1.73 & 1.79 & 1.73 & 1.52 & 1.52 & 1.51 & 1.52 & 1.14 & 1.14 & 1.19 & 1.14 \\
\hline & $(4,4)$ & 2.25 & 2.13 & 2.32 & 2.55 & 2.02 & 1.67 & 1.94 & 2.37 & 1.11 & 1.27 & 1.19 & 1.07 \\
\hline \multicolumn{14}{|c|}{$\sigma_{1}=1, \sigma_{2}=3$} \\
\hline \multirow{5}{*}{1} & $(5,5)$ & 2.53 & 2.61 & 2.22 & 2.43 & 2.37 & 2.59 & 2.08 & 2.11 & 1.06 & 0.98 & 1.04 & 1.12 \\
\hline & $(5,10)$ & 3.13 & 2.76 & 2.58 & 3.07 & 2.75 & 2.43 & 1.98 & 2.86 & 1.09 & 1.09 & 1.25 & 1.03 \\
\hline & $(15,15)$ & 6.04 & 4.73 & 3.84 & 5.60 & 5.44 & 3.38 & 3.76 & 5.37 & 1.10 & 1.39 & 1.01 & 1.04 \\
\hline & $(15,20)$ & 6.69 & 4.78 & 4.00 & 6.31 & 5.87 & 2.86 & 3.16 & 6.14 & 1.12 & 1.64 & 1.24 & 1.01 \\
\hline & $(20,20)$ & 7.88 & 5.27 & 4.42 & 7.55 & 7.01 & 3.04 & 3.20 & 7.33 & 1.12 & 1.73 & 1.37 & 1.02 \\
\hline \multirow{5}{*}{5} & $(2,2)$ & 1.39 & 1.39 & 1.39 & 1.39 & 1.17 & 1.17 & 1.17 & 1.17 & 1.19 & 1.19 & 1.19 & 1.19 \\
\hline & $(2,3)$ & 1.51 & 1.51 & 1.54 & 1.51 & 1.32 & 1.32 & 1.38 & 1.32 & 1.13 & 1.13 & 1.10 & 1.13 \\
\hline & $(2,4)$ & 1.63 & 1.56 & 1.65 & 1.58 & 1.32 & 1.44 & 1.25 & 1.29 & 1.21 & 1.06 & 1.28 & 1.20 \\
\hline & $(3,4)$ & 1.93 & 1.83 & 1.88 & 1.91 & 1.71 & 1.45 & 1.48 & 1.72 & 1.12 & 1.24 & 1.25 & 1.10 \\
\hline & $(4,4)$ & 2.17 & 2.10 & 2.10 & 2.43 & 1.87 & 1.54 & 1.75 & 2.19 & 1.16 & 1.36 & 1.20 & 1.11 \\
\hline \multirow{3}{*}{10} & $(2,2)$ & 1.38 & 1.38 & 1.38 & 1.38 & 1.07 & 1.07 & 1.07 & 1.07 & 1.29 & 1.29 & 1.29 & 1.29 \\
\hline & $(3,3)$ & 1.80 & 1.80 & 1.74 & 1.80 & 1.41 & 1.41 & 1.42 & 1.41 & 1.27 & 1.27 & 1.22 & 1.27 \\
\hline & $(4,4)$ & 2.10 & 2.01 & 1.99 & 2.31 & 1.79 & 1.18 & 1.66 & 2.08 & 1.16 & 1.69 & 1.19 & 1.10 \\
\hline
\end{tabular}




\subsection{Comparison of SRS with the RSS and its Modifications}

According to $R E_{1}, R E_{2}, R E_{3}$ and $R E_{4}$ values, the ML estimators of the system reliability $R$ based on RSS and its modifications are more efficient than their counterpart estimator based on SRS. In terms of $R E_{5}, R E_{6}, R E_{7}$ and $R E_{8}$, the MML estimators of $R$ based on all four methods are superior to the MML estimator of $R$ based on SRS.

In the case of $r_{x}=r_{y}=1, \sigma_{1}=\sigma_{2}=1$ and $\left(m_{x}, m_{y}\right)$ are small, and RSS and its modifications are more efficient than SRS. However, efficiencies of the RSS and its modifications are close to each other. When $\left(m_{x}, m_{y}\right)$ are moderate or large all methods are more efficient than SRS, but ERSS is the least efficient among the others. When $\sigma_{1}=$ $1, \sigma_{2}=2$ and $\sigma_{1}=1, \sigma_{2}=3$, the ML and MML estimators of $R$, based on ERSS and MRSS, are less efficient than their counterparts based on RSS and PRSS.

It is clear from the simulation results presented in Table 1 that in the case of $r_{x}=r_{y}=5$ and $r_{x}=r_{y}=10$ the set sizes are vary between $(2,2)$ and $(4,4)$. The efficiencies of RSS and its modifications are extremely similar to each other. It should be realized that $R E \mathrm{~s}$ are not particularly affected by an increase in the number of cycles. As in a small number of cycles, the proposed estimators are more efficient than their counterpart estimator, based on SRS.

Simulation studies are repeated for several other shape parameters $p$. However, it can be seen from Table 1 that $p$ values do not have an influence on the efficiencies of the estimators of system reliability $R$.

\subsection{Comparison of ML and the MML Estimators based on RSS and its Modifications}

ML and MML estimators of $R$ based on RSS and its modifications, are compared using the $R E_{9}, R E_{10}, R E_{11}$ and $R E_{12}$. When $r_{x}=r_{y}=1$ and $\sigma_{1}=\sigma_{2}=1$ efficiencies of the ML and MML estimators of $R$, based on PRSS, are more or less the same as the set sizes $\left(m_{x}, m_{y}\right)$ increases. $\hat{R}_{M M L, E R S S}$ is more efficient than $\hat{R}_{M L, E R S S}$. In addition, $\hat{R}_{M L, M R S S}$ performs better than $\hat{R}_{M M L, M R S S}$.

When the number of cycles $r_{x}=r_{y}=5$ and $r_{x}=r_{y}=10$ and the set sizes $\left(m_{x}, m_{y}\right)$ are small the ML estimators are more efficient than the MML estimators as excepted.

\section{Conclusion}

In this paper, we obtain the ML and the MML estimators of $R$, based on ERSS, MRSS and PRSS, where $X$ and $Y$ are independent Weibull random variables. We then compare the performances of the proposed estimators and their counterparts based on RSS to the ML and the MML estimators of $R$ based on SRS. It is clear from the simulation results that the use of RSS and its modifications are highly efficient when compared to SRS for estimating the system reliability $R$ using the ML and the MML estimators.

If we are interested in comparing the estimators, with respect to their efficiency, then the ML and the MML estimators based on RSS are preferable. On the other hand, if our focus 
is to reduce the ranking error, then we should use the estimators based on ERSS, MRSS and PRSS.

\section{References}

1. Abu-Dayyeh, W. A., Al-Subh, S. A. and Muttlak, H. A. (2004). Logistic parameters estimation using simple random sampling and ranked set sampling. Appl. Math. Comput., 150, 543-554.

2. Abu-Dayyeh, W. A., Assrhani, A. and Ibrahim, K. (2013). Estimation of the shape and scale parameters of Pareto distribution using ranked set sampling. Stat. Pap., 54(1), 207-225.

3. Akgül, F. G. (2015). Robust estimation of system reliability in stress-strength model using ranked set sampling. Ph.D. diss., Ankara University, Ankara, Turkey.

4. Akgül, F. G. and Şenoğlu, B. (2017). Estimation of $\mathrm{P}(\mathrm{X}<\mathrm{Y})$ Using Ranked Set Sampling for Weibull Distribution. QTQM, 14(3), 296-309.

5. Akgül, F. G. and Şenoğlu, B. (2014). Estimation of the Reliability in the StressStrength Models under the Modifications of the Ranked Set Sampling: Weibull Distribution. $11^{\text {th }}$ International Conference on Ordered Statistical Data, 22-23, Bedlewo, Poland.

6. Al-Nasser, A. D. (2007). L ranked set sampling: A generalization procedure for robust visual sampling. Commun. Stat. Simulat., 36, 33-43.

7. Al-Saleh, M. F. and Al-Kadiri, M. A. (2000). Double ranked set sampling. Stat. Prob. Lett., 48(2), 205-212.

8. Bhoj, D. S. (1997). Estimation of parameters of the Extreme Value distribution using ranked set sampling. Commun. Stat. Theory., 26(3), 653-667.

9. Chen, Z., Bai, Z. D. and Sinha, B. K. (2004). Ranked Set Sampling: Theory and Applications. Springer, New York, NY, USA.

10. Church, J. D. and Harris, B. (1970). The estimation of reliability from stressstrength relationship. Technometrics, 12, 49-54.

11. Constantine, K., Karson, M. and Tse, S. K. (1986). Estimators of $P(Y<X)$ in the gamma case. Commun. Stat. Simulat., 15, 365-388.

12. Dastbaravarde, A. and Zamanzade, E. (2017). On estimation of $P(X>Y)$ based on judgment post stratification. To appear in Statistical Papers.

13. Dong, X., Zhang, L. and Li, F. (2013). Estimation of reliability for exponential distributions using ranked set sampling with unequal samples. QTQM, 10(3), 319328.

14. Helu, A., Abu-Salih, M. and Alkam, O. (2010). Bayes estimation of Weibull distribution parameters using ranked set sampling. Commun. Stat. Theory., 39, 2533-2551.

15. Kotz, S., Lumelskii, Y. and Pensky, M. (2003). The Stress-Strength Model and Its Generalizations. World Scientific Press, Singapore. 
16. Kundu, D. and Gupta, R. D. (2006). Estimation of $P(Y<X)$ for Weibull distributions. IEEE Trans. Reliab., 55, 2, 270-280.

17. Lam, K., Sinha, B. K. and Wu, Z. (1994). Estimation of parameters in a twoparameter exponential distribution using ranked set sampling. Ann. Inst. Statist. Math. 46(4), 723-736.

18. Mahdizadeh, M., and Zamanzade, E. (2016a). Kernel-based estimation of $P(X>Y)$ in ranked set sampling. Statistics and Operations Research Transactions (SORT), 40(2), 243-266.

19. Mahdizadeh, M. and Zamanzade, E. (2016b). A new reliability measure in ranked set sampling. Statistical Papers, doi: 10.1007/s00362-016-0794-3.

20. Mahdizadeh. M, and Zamanzade, E. (2017). Reliability estimation in multistage ranked set sampling. REVSTAT: A Statistical Journal, 15 (4), 565-581.

21. McIntyre, G. A. (1952). A method for unbiased selective sampling, using ranked sets. Aust. J. Agr. Res., 3, 385-390.

22. Muttlak, H. A. (1997). Median ranked set sampling. J. Appl. Stat. Sci., 6(4), 245255.

23. Muttlak, H. A. (2003). Modified ranked set sampling methods. Pak. J. Stat., 19(3), 315-323.

24. Muttlak, H. A., Abu-Dayyeh, W. A., Saleh, M. F. and Al-Sawi, E. (2010). Estimating $P(Y<X)$ using ranked set sampling in case of the exponential distribution. Commun. Stat. Theory., 39, 1855-1868.

25. Noughabi, H. A. (2017). Efficiency of ranked set sampling in tests for normality. Journal of Statistical Computation and Simulation, 87(5), 956-965.

26. Samawi, H. M., Ahmed, M. S. and Abu-Dayyeh, W. A. (1996). Estimating the population mean using extreme ranked set sampling. The Biometrical J., 38(5), 577-586.

27. Samawi, H. M., Rochani, H., Linder, D. and Chatterjee, A. (2017). More efficient logistic analysis using moving extreme ranked set sampling. Journal of Applied Statistics, 44(4), 753-766.

28. Sengupta, S. and Mukhuti, S. (2008a). Unbiased estimation of $P(X>Y)$ for exponential populations using order statistics with application in ranked set sampling. Commun. Stat. Theory., 37, 898-916.

29. Sengupta, S. and Mukhuti, S. (2008b). Unbiased estimation of $P(X>Y)$ using ranked set sample data. Statistics: J. Theor. Appl. Stat., 42(3), 223-230.

30. Shaibu, A. -B. and Muttlak, H. A. (2002). A comparison of the maximum likelihood estimators under ranked set sampling some of its modifications. Appl. Math. Comput., 129, 441-453.

31. Shaibu, A. -B. and Muttlak, H. A. (2004). Estimating the parameters of the normal, exponential and gamma distributions using median and extreme ranked set samples. Statistica, 64(1), 75-98. 
32. Takahasi, K. and Wakimoto, K. (1968). On unbiased estimates of the population mean based on the sample stratified by means of ordering. Ann. I. Stat. Math., 20(1), 1-31.

33. Tiku, M. L. (1967). Estimating the mean and standard deviation from a censored normal sample. Biometrika, 54, 155-165.

34. Tiku, M. L. (1968). Estimating the parameters of log-normal distribution from a censored sample. J. Amer. Stat. Assoc., 63, 134-140.

35. Tiku, M. L. and Akkaya, A. D. (2004). Robust Estimation and Hypothesis Testing. New Age International (P) Limited, Publishers, New Delhi.

36. Tong, H. (1977). On the estimation of $P(Y<X)$ for exponential families. IEEE Trans. Reliab., 26, 54-56.

37. Vaughan, D. C. and Tiku, M. L. (2000). Estimation and hypothesis testing for a non-normal bivariate distribution and applications. J. Math. Comput. Model., 32, 53-67.

38. Zamanzade, E., and Al-Omari, A.I. (2016). New ranked set sampling for estimating the population mean and variance. Hacettepe Journal of Mathematics and Statistics, 45(6), 1891-1905. 